\title{
Ekman Drift in Homogeneous Water
}

\author{
W. Krauss \\ Institut für Meereskunde an der Universität Kiel, Kiel, Germany
}

\begin{abstract}
Measurements made with satellite-tracked buoys drogued in different layers between the sea surface and 30-m depth under homogeneous winter conditions in the North Sea allow analysis of the Ekman currents under a large variety of wind conditions. The experiment lasted from November 20, 1991, until February 29, 1992. The first 4 weeks of this period, during which the buoys stayed close together, are used to determine the Ekman stresses. The total current field is a superposition of barotropic currents due to sea level variations and Ekman currents. The classical Ekman theory is not able to describe properly the observed deflection of the currents to the right of the wind direction and their decay with depth. This deflection is $10^{\circ}$ near the sea surface and increases to approximately $50^{\circ}$ in $25-\mathrm{m}$ depth. The relation between wind stress and the stress field in the interior of the water is given by a tensor, which describes the rotation and the variation of the stress with increasing depth. The concept of eddy viscosity is applicable, if a viscosity tensor is used to relate stress and vertical shear. The viscosity tensor is a function of the vertical coordinate only and is independent from the wind stress. It shows maximum values in $15-$ to 20 -m depth and may be due to Langmuir circulation cells. Further studies are needed to determine the physics of this tensor. Its magnitude in the interior of the mixed layer exceeds $1000 \mathrm{cgs}$ units. Consequently, Ekman currents are weak and may not be the dominant currents within the mixed layer.
\end{abstract}

\section{INTRODUCTION}

The deflection of the ice drift to the right of the wind direction was first recognized and interpreted by Nansen [1902] during the drift of the Fram in 1893-1896. He concluded that the same must hold for wind-driven ocean currents. Their first mathematical description was given by Ekman [1902, 1905], who considered both infinite and finite depth and included Fredholm's solution for the unsteady case. In his classical paper he wrote [Ekman, 1905, p. 39], "The magnitude of D or 'Depth of frictional influence' is the key which must be found, before the theory here given can be made fully applicable." This depth is crucial for the Ekman spiral and depends on the unknown eddy viscosity (we denote eddy viscosity by $\mu$ and kinematic eddy viscosity by $\nu$; dimensions are grams per centimeter per second and square centimeter per second, respectively. Both quantities are used in the literature).

The major results of Ekman's theory are as follows.

1. Under stationary conditions and constant eddy viscosity, the current will be deflected to the right of the wind direction by $45^{\circ}$ at the surface (northern hemisphere) and rotates further with depth. If $\mu=10 \mathrm{~cm}^{2} \mathrm{~s}^{-1}$, the Ekman depth, where the current is opposite to the surface current, is only $15 \mathrm{~m}$ in midlatitudes. For $\mu=1000 \mathrm{~cm}^{2} \mathrm{~s}^{-1}$ it increases to $140 \mathrm{~m}$.

2. The speed of the current is inversely proportional to $\mu^{1 / 2}$. A water parcel at the surface moves approximately 32 $\mathrm{km} \mathrm{d}^{-1}$ for $\mu=10$ and only $3.2 \mathrm{~km} \mathrm{~d}^{-1}$ for $\mu=1000$.

3. The speed decreases with depth according to exp $\left(-1 / \mu^{1 / 2}\right)$. Using $\mu=10$, the speed in $10 \mathrm{~m}$ depth is only $1 \%$ of the surface speed; for $\mu=1000$ this value is $64 \%$.

4. Inertial waves which describe the adaptation of the current to the balance of stress and Coriolis force in the nonstationary case, decrease with depth, are delayed in time

Copyright 1993 by the American Geophysical Union.

Paper number 93JC01898.

0148-0227/93/93JC-01898\$05.00 according to $\exp \left(-\rho z^{2} / 4 \mu t\right)$, and are convoluted with the time-dependent wind stress.

Numerous attempts have been made to verify the Ekman theory, which turned out to be difficult owing to the superposition of geostrophic or gradient currents and the variability of the current system under variable wind conditions. With respect to older literature, we refer to the summaries given by Defant [1960] and Huang [1979]. Generally, deflections of the current to the right of the wind are observed; however, the angle of deflection varies over a large range. Observations in shallow water are additionally complicated by bottom topography or coastlines. Stratification of the upper layers yields further complexity. With respect to eddy viscosity $\mu$, relations to the wind speed $W$ have been proposed already by Ekman [1905] and Thorade [1914], such as $\mu=1.03 W^{3}$ for $W<6 \mathrm{~m} \mathrm{~s}^{-1}$ and $\mu=4.3 W^{2}$ for $W$ $>6 \mathrm{~m} \mathrm{~s}^{-1}$, where $\mu$ is given in cgs units. This yields $\mu=28$ for $W=3 \mathrm{~m} \mathrm{~s}^{-1}$ and $\mu=1720$ for $W=20 \mathrm{~m} \mathrm{~s}^{-1}$. Generally, we can quote Defant [1960, p. 422]: "All these formulae are of course only approximations, since at the present time systematic current measurements from which accurate values could be derived are not available." However, more recent measurements have shed some more light on the problem.

A clear Ekman spiral was observed by Gonella [1968] from measurements during a 9-day period in deep water in the Mediterranean. The corresponding eddy viscosity responsible for the observed spiral was $1200 \mathrm{cgs}$ units, yielding an Ekman depth of $155 \mathrm{~m}$. Even larger values $\left(5300 \mathrm{~cm}^{2} \mathrm{~s}^{-1}\right)$ were obtained by Stacey et al. [1986] in the Strait of Georgia, British Columbia, where the water depth was $370 \mathrm{~m}$. Measurements lasted from June 1984 to January 1985 and covered 15 depth intervals. The area is known for having strong tidal currents. The deduced Ekman spiral, described by the first empirical orthogonal function, showed a deflection of only $20^{\circ}$ in $20-\mathrm{m}$ depth but showed a well-pronounced rotation down to $300 \mathrm{~m}$. The decrease in speed was less than that predicted by Ekman's theory. Also, the observations based on dye dilfusion experiments such as those of lchiye 
[1967] or Katz et al. [1965] clearly indicate the existence of an Ekman-type spiral, but the shape often differed markedly. Weller [1981] arrived at the conclusion that the angle of deflection in his measurements off California was best modeled when the eddy viscosity was of the order of $500 \mathrm{cgs}$ units, but the amplitudes were best described when the eddy viscosity was of the order of $50 \mathrm{cgs}$ units. Similar results were obtained during the Long-Term Upper Ocean Study (LOTUS) [Price et al., 1987].

Eddy viscosity as caused by turbulent motion appears to be critically dependent on stratification. This holds both for the seasonal thermocline and for the diurnal cycle. If heat and momentum are mixed in a similar way, the penetration depth for the momentum flux shoals during midday with the penetration depth for the heat flux. Thus the velocity is surface intensified during daytime under fair weather conditions [Price et al., 1986]. For cases like this, Kondo et al. [1979] computed eddy viscosities of the order of 10 at the surface which increased during nighttime to the order of 1000 in 10-m depth and remained about $50 \mathrm{cgs}$ units below. As a consequence, the clockwise deviation of the current from the wind direction was larger during the day than during the night. Additionally, a diurnal jet may develop [Price et al., 1986]. These complications can be avoided by measurements under winter conditions in areas where the daily cycle is less pronounced and where the mixed layer reaches deep enough to guarantee homogeneity. Under these conditions, McNally and White [1985] obtained deflections of $20^{\circ}$ in the North Pacific between $35^{\circ} \mathrm{N}$ and $38^{\circ} \mathrm{N}$. Moreover, the deflection was the same at $30-\mathrm{m}, 60-\mathrm{m}$, and $90-\mathrm{m}$ depth.

However, even in homogeneous water the parameterization of the eddy viscosity is still an open problem. Being a dynamic property of the flow the eddy viscosity plays the central role in describing details of the Ekman layer. Several questions can be raised:

1. Is eddy viscosity wind or wave dependent? Besides the relation by Thorade [1914], Neumann and Pierson [1964] proposed $\mu=0.1825 \times 10^{-4} W^{5 / 2}$ ( $W$ in centimeters per second). According to Leibovich and Radhakrishnan [1977], $\mu=2.84 \times 10^{-5} W^{3 / 2}$ should hold, and Ichiye [1967] suggested $\mu=0.028 H / T$, where $H$ is the wave height and $T$ is the wave period. Further relations are listed by Huang [1979]. If the turbulence in the near-surface layer is created by wind stirring or wave breaking, an increase of $\mu$ with $W$ seems plausible, but none of the formulae given above is sufficiently supported by measurements.

2. Is eddy viscosity approximately constant with depth, or does it increase or decrease with depth? Constant eddy viscosity is necessary in order to obtain a deflection of the current of $45^{\circ}$ at the surface. If eddy viscosity increases with depth, the deflection becomes less than $45^{\circ}$ and can be reduced to $10^{\circ}$ as in the model of Madsen [1977]. On the other hand, decreasing eddy viscosity with depth yields larger deflections than $45^{\circ}$.

A linear increase with depth of the eddy viscosity is based on an inverted atmospheric boundary layer model where the velocity profile is given by the logarithmic law. The constant stress assumption, upon which the logarithmic law is based, results in $\mu=\rho k W_{*} z$, in which $W_{*}$ is the frictional velocity and $k$ is the Kármán constant.

On the other hand, if wind stress and wave braking were the source of turbulence, one would expect that its intensity would decrease with depth and that eddy viscosity should be a decreasing function of depth.

3. Are eddy viscosities of order 100 or $1000 \mathrm{cgs}$ units typical for the ocean? If the formulae given above that relate $\mu$ and $W$ have any reliability, $\mu$ would exceed the order of $1000 \mathrm{cgs}$ units frequently during winter time in mid and high latitudes. Eddy viscosities of the order of 1000 not only are required to interpret Ekman deflections in some cases but were also needed to balance advection at a front [Flament et al., 1985]. However, such large values are in contrast to all general circulation models which use values of the order of 10 in the uppermost layer. Consequently, the entire Ekman transport is usually confined to the uppermost model layer and the meridional heat transport [Böning and Herrmann, 1993] is based on the temperature of this layer. If $\mu$ were of the order of 1000 , the entire upper $150 \mathrm{~m}$ would contribute to the Ekman transport with the temperatures of the corresponding layers. Furthermore, the transport within these layers were in different directions according to the Ekman spiral.

In order to obtain more information on the Ekman spiral and on eddy viscosity, an experiment has been performed in winter 1991-1992 in the central North Sea with satellitetracked buoys drogued in different depths within the upper $30 \mathrm{~m}$ of the water column. The location has been chosen in order to fulfill one of the prerequisites of the theory: homogeneous conditions. In the following sections we describe the data set (section 2), the stress field and its statistics (sections 3 and 4), the Ekman stresses (section 5) and the viscosity tensor (section 6). The remaining part of the stress which is not related to the wind will be interpreted in section 7. The results are summarized and discussed in sections 8 and 9 .

\section{Data Set}

Ten satellite-tracked buoys at five different levels within the upper $30 \mathrm{~m}$ were deployed on November 20, 1991, at $57^{\circ} \mathrm{N}, 2^{\circ} \mathrm{E}$, in the central North Sea. The position is shown in Figure 1. Water depth is $80 \mathrm{~m}$. The site was chosen because the central North Sea is homogeneous during winter. Thus we avoid the influence of stratification on the Ekman spiral. Furthermore, the wind field in the area is rather well known.

The currents in the area are known to be weak, variable, and wind-dependent. The buoys are cylinders of $2.6-\mathrm{m}$ length and $11-\mathrm{cm}$ diameter, drogued by a holey sock of $10-\mathrm{m}$ length with aballast weight of $7 \mathrm{~kg}$ at the bottom. Their drift performance was described by Bitterman and Hansen [1986]. Drogues covered the ranges 3-13 m, 7-17 m, 12-22 $\mathrm{m}, 17-27 \mathrm{~m}$, and 22-32 $\mathrm{m}$. Two buoys were deployed at each level, but owing to heavy traffic in the area several losses occurred and only one buoy was used at each level for the final analysis. For the interpretation of the results, the data were attached to model depths of 7.5, 12.5, 17.5, 22.5, and $27.5 \mathrm{~m}$ (Figure 2). The measurements extend from November 20, 1991, to February 29, 1992, but in this article only the first 4 weeks are analyzed. During this period the buoys stayed close together, allowing us to derive relations between wind stress and the stress in the interior without getting complications from spatial inhomogeneities. Thus the data described cover the time range November 20, 0000 UT, to December 19, 1900 UT, or 716 hours.

Positions via Argos were received 6--8 times per day. Data 


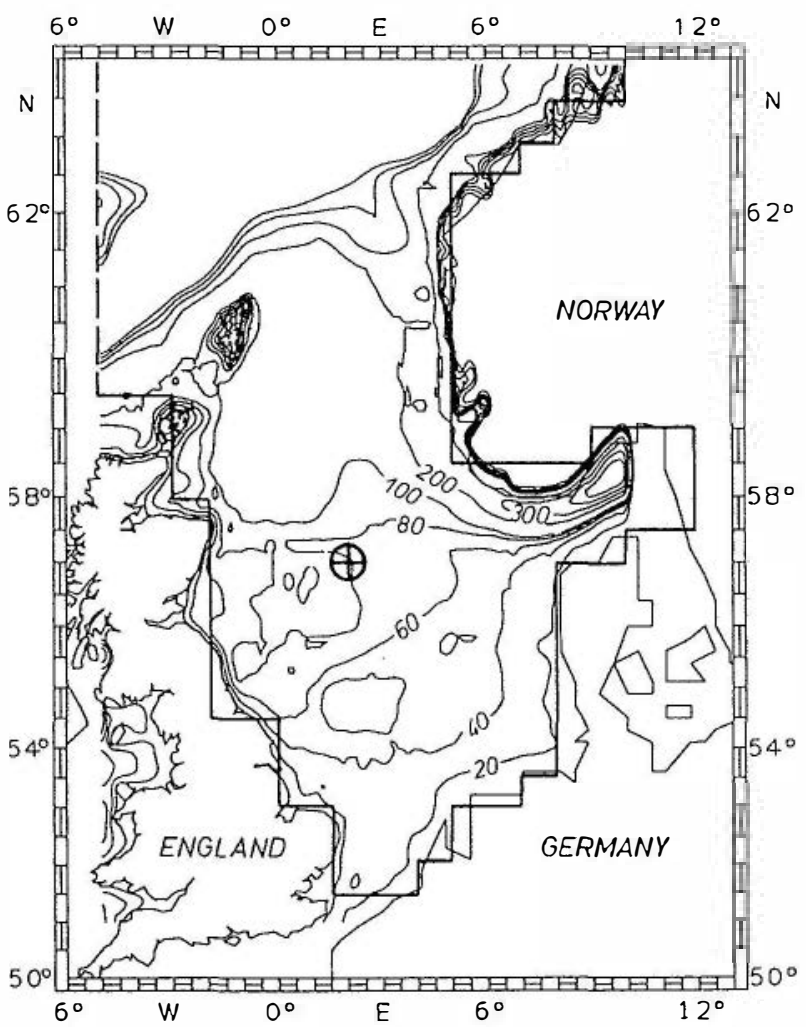

Fig. 1. Area of buoy deployment in the North Sea (cross hairs) and boundaries of the barotropic model.

for which the interval between consecutive positions was less than 3 hours were omitted. The final time series were interpolated by cubic splines to 1 -hour intervals. Figure 3 shows an example of the north component of the drift during these 716 hours in $7.5-\mathrm{m}$ depth. The short periodic fluctuations are semidiurnal tidal fluctuations and inertial waves. The tidal currents are typically $10-15 \mathrm{~cm} \mathrm{~s}^{-1}$ in the northsouth direction and $5 \mathrm{~cm} \mathrm{~s}^{-1}$ in east-west direction. To further reduce tidal fluctuations (12.4 hours) and inertial waves ( 14.2 hours), the data have been smoothed by computing running means over 13 hours. This reduces tidal

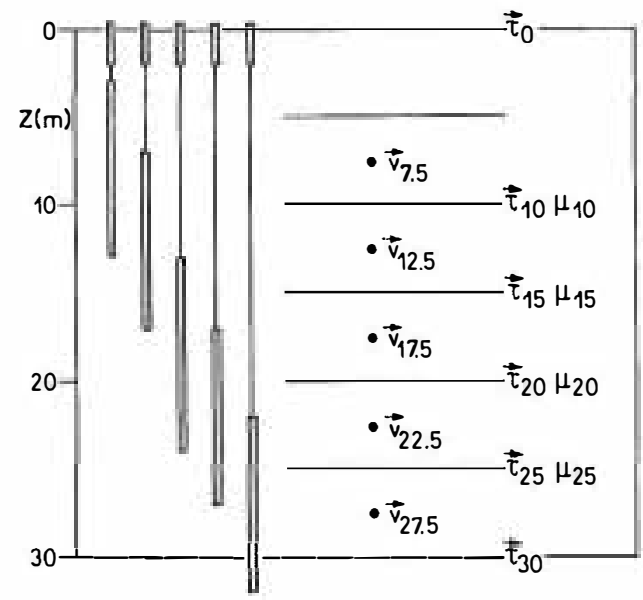

Fig. 2. Buoy configuration and scheme for the stress computations.

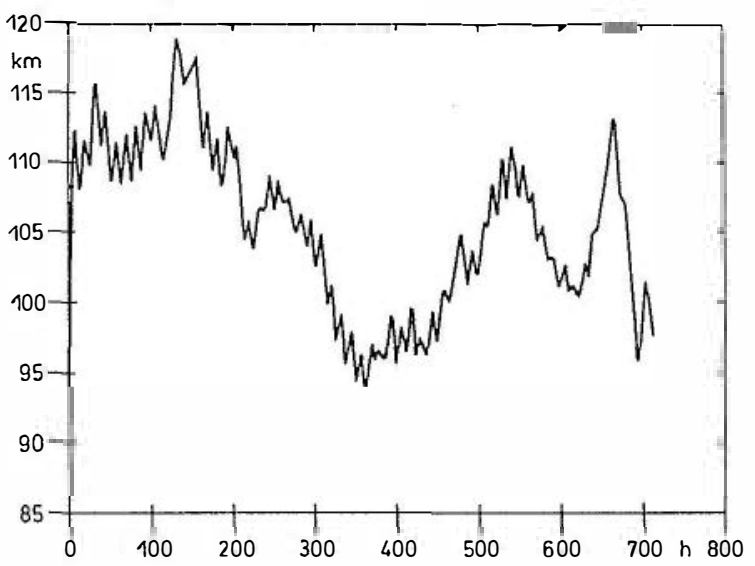

Fig. 3. Spline interpolated north component of the drift at $7.5-\mathrm{m}$ depth.

currents to $5 \%$ and inertial currents to $10 \%$ of their original amplitudes. These data are then used to compute velocities.

Meteorological data (wind velocity at $10 \mathrm{~m}$ and surface pressure) were made available by the German weather service from the Europe model on a $50 \times 50 \mathrm{~km}$ grid at 6-hourly intervals. During the first 10 days, wind was from south reaching $18 \mathrm{~m} \mathrm{~s}^{-1}$, then followed a period with variable and light winds. During the last 10 days, westerly winds up to $18 \mathrm{~m} \mathrm{~s}^{-1}$ prevailed. The wind stress $\tau_{0}$ for the location of deployment computed according to the relations of Large and Pond [1981] is depicted in Figure 4. The components exceed $5 \mathrm{~g}\left(\mathrm{~cm} \mathrm{~s}^{2}\right)^{-1}$ during the stormy periods and allow to study the Ekman response under a large variety of conditions.

Figure 5 shows the total drift of the buoys. They start at $\mathrm{km} x=60, y=110$ in our arbitrary coordinate system. The dots on the trajectories indicate the position after 500 hours, which include the southerly storms during the first 10 days and the relatively calm period during the remaining 10 days. During the final 10 days of strong westerly winds they all move southeast. As the North Sea is homogeneous during this time of the year, only barotropic currents due to sea level inclinations and Ekman currents are possible. As mentioned in the introduction, eddy viscosities of the order of $1000 \mathrm{cgs}$ units are required to reduce the Ekman drifts to about $3 \mathrm{~km} \mathrm{~d}^{-1}$. All drifters are displaced approximately 40 $\mathrm{km}$ in 30 days, the lowest one even less. If Ekman's theory were valid with $\mu<100$ cgs units, the buoys would have been separated by several hundred kilometers during the stormy periods. The observed trajectories are a first indication that a constant eddy viscosity less than $100 \mathrm{~cm}^{2} \mathrm{~s}^{-1}$ is inadequate to describe the response of the upper layers.

\section{The Apparent Stress Field}

The following analysis is based on the velocities computed from the drift components. They are depicted in Figures $6 a$ and $6 b$ for the east $(u)$ and north component $(v)$, respectively. The amplitudes of the fluctuations are of similar order at all depths, and the mean value is approximately the same.

These velocities are used to compute the stress field in the upper $30 \mathrm{~m}$ of the water column. As the velocities exceed seldom $10 \mathrm{~cm} \mathrm{~s}^{-1}$, the topography in the area is smooth, and the wind field is large scale, neither in the barotropic 


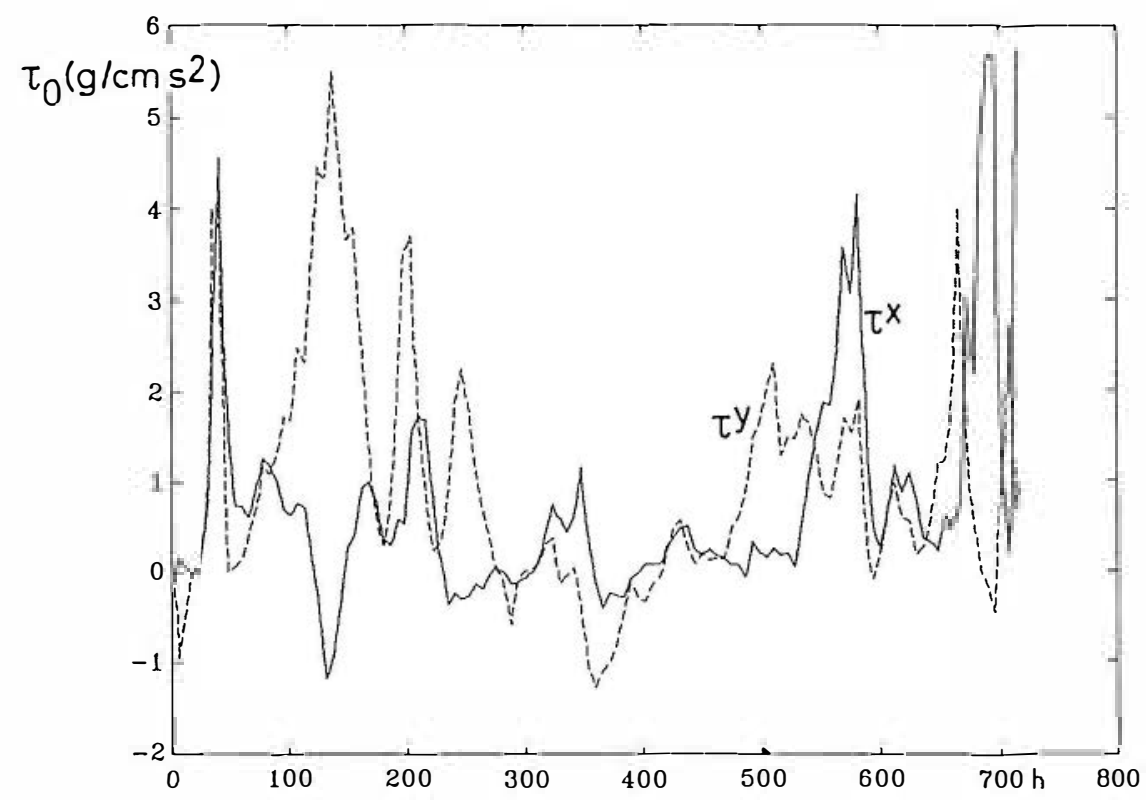

Fig. 4. Wind stress at the position of deployment during November 20 to December 20, 1991. Solid line, east component; dashed line, north component.

currents nor in the Ekman currents should the nonlinear terms in the equation of motion play any role.

According to Figure 2 and on the basis of $n$ levels,

$$
\begin{aligned}
& \frac{\partial u_{n}}{\partial t}-f v_{n}=-g \frac{\partial \zeta}{\partial x}+\frac{\tau_{n-1}^{x}-\tau_{n}^{x}}{h_{n} \rho} \\
& \frac{\partial v_{n}}{\partial t}+f u_{n}=-g \frac{\partial \zeta}{\partial y}+\frac{\tau_{n-1}^{y}-\tau_{n}^{y}}{h_{n} \rho}
\end{aligned}
$$

we can compute consecutively the stresses at $10-\mathrm{m}, 15-\mathrm{m}$, 20-m, 25-m, and 30-m depth from the observed velocities $u_{n}$, $v_{n}$, the layer thickness $h_{n}$, and the wind stress $\tau_{0}$ at the

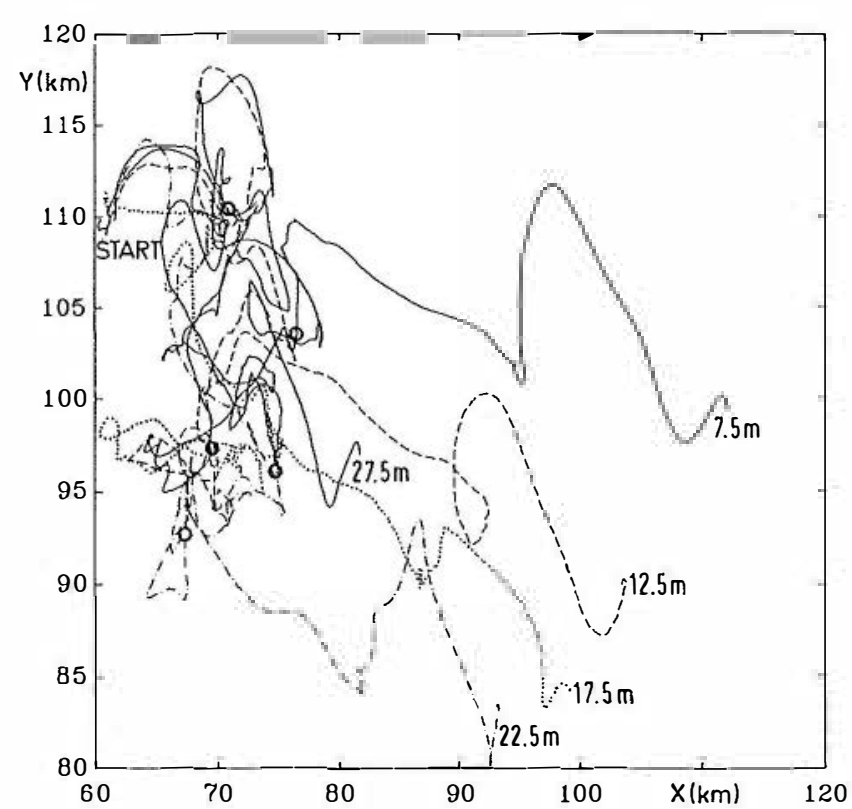

Fig. 5. Drift of buoys at five levels from November 20 to December 20,1991. The starting point is at $\mathrm{km} x=60, y=110$. The dots mark the positions after 500 hours of drift. surface, if we know the inclinations of the sea surface, $\zeta_{x}$, $\zeta_{y}$. These were obtained from a vertically integrated model of the North Sea, based on (1), (2), and the continuity equation. The model area is shown in Figure 1. Grid distances are $1 / 2^{\circ}$ in the $x$ (east) direction and $1 / 4^{\circ}$ in the $y$ (north) direction. On average, this corresponds to $\Delta x=30$ $\mathrm{km}, \Delta y=28 \mathrm{~km}$. The model is closed in the English Channel and in the Kattegat but has open boundaries at $64^{\circ} \mathrm{N}$ and along $5^{\circ} \mathrm{W}$. At open boundaries the normal component of the currents is supposed to be geostrophic. In order to reduce the computational effort, the wind field for these calculations was computed from the surface pressure distribution by approximating the pressure distributions by quadratic surfaces. The geostrophic wind was then reduced to $75 \%$ and turned $15^{\circ}$ to the left in order to obtain the surface wind [Luthardt and Hasse, 1983]. By this means, only six coefficients are needed at each time step $(d t=300 \mathrm{~s})$ instead of the entire input field at approximately 1800 grid points. Bottom friction was finally chosen as $\tau_{-H}=r v$ with $r=$ $500 / H(x, y)^{2} ; H$ is the water depth, and $v$ is the vertically integrated current. The stresses obtained are called "apparent stresses" because they may include errors due to (1) the drift velocities $u$ and $v$ (comparison of drifters at the same depth shows differences of $0.4 \mathrm{~cm} \mathrm{~s}^{-1}$ in the mean velocity and $0.6 \mathrm{~cm} \mathrm{~s}^{-1}$ in the standard deviation), (2) our approximation of the real pressure fields by quadratic surfaces and the deduced wind velocities, and (3) the computed inclination of the sea surface, which depends on the parameterization of the wind stress and slightly on the bottom friction. Inclinations become larger if the bottom stress is reduced. However, as only the difference $\tau_{0}-\tau_{-H}$ is important as a driving force in the vertically integrated equations, the drag coefficient in $\tau_{0}$ is much more important than the bottom stress. For a wind of $15 \mathrm{~m} \mathrm{~s}^{-1}$ the drag coefficients given by various authors differs by $25 \%$. If a dependency on the significant wave height is included, the difference can even increase much more [Blake, 1991]. We therefore adopted a relation for $r$ used previously in storm surge models [Fis- 

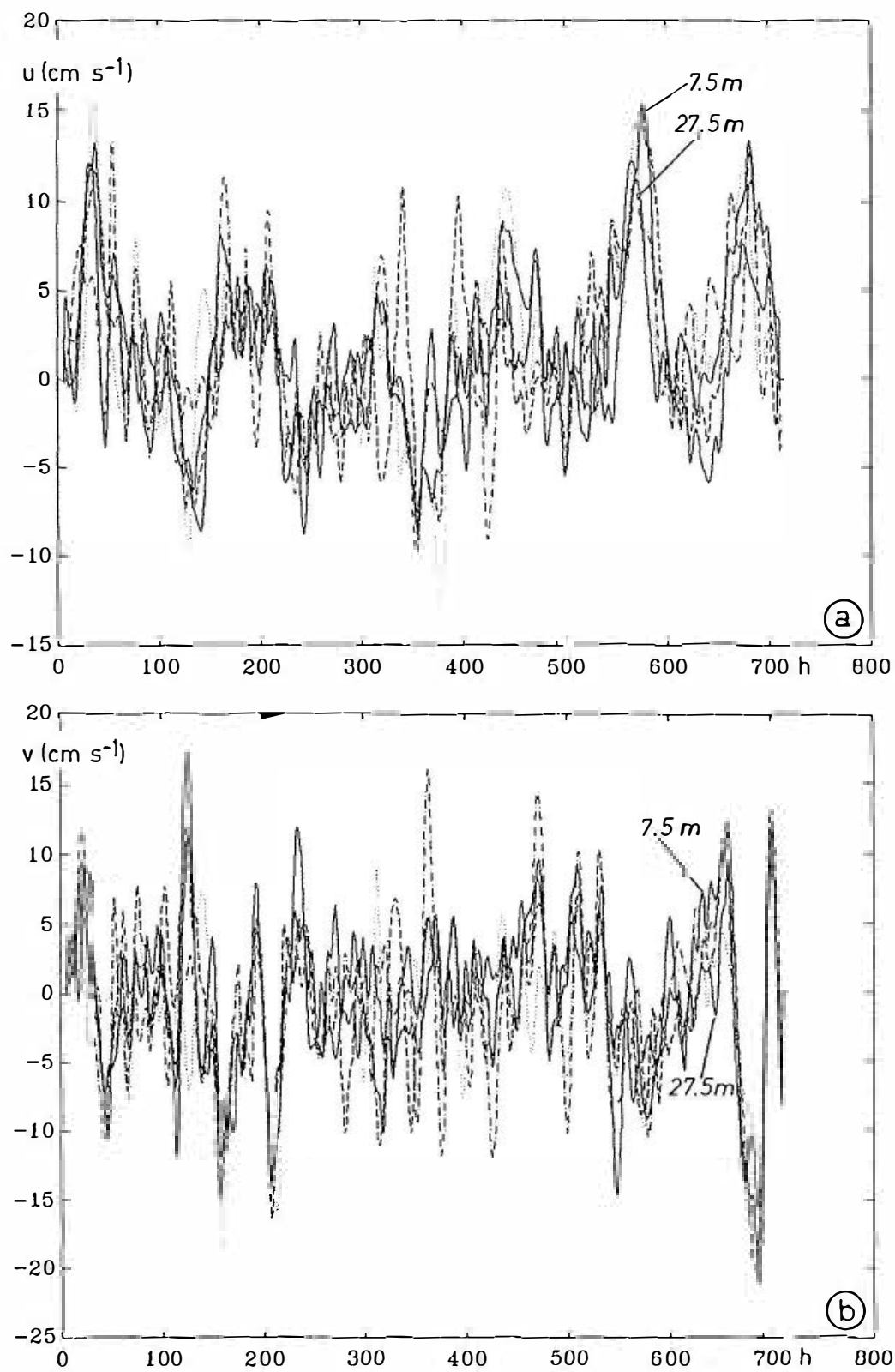

Fig. 6. (a) East and $(b)$ north components of the drift velocities at depths of $7.5 \mathrm{~m}$ (solid line), $12.5 \mathrm{~m}$ (dashed line), $17.5 \mathrm{~m}$ (dotted line, $22.5 \mathrm{~m}$ (dashed-dotted line), and $27.5 \mathrm{~m}$ (lower solid line).

cher, 1959]. Furthermore, external surges from outside the model area may travel into the North Sea and produce additional inclinations of the sea surface.

These errors accumulate at larger depth, as the computation of the apparent stresses starts at the uppermost level. Errors are transferred to all consecutive levels.

The apparent stresses are depicted in Figure 7 together with the wind stress. These figures show that the stresses are similar in all depth and that the wind stress is much smoother. An enhanced part of Figure $7 b$ is shown in Figure 8 for the $y$ components of the stress during the first 200 hours. The main features are that (1) the stress decreases with depth, as is to be expected if the wind stress and breaking waves are the energy source of turbulence which determines the stress, and (2) many fluctuations in the stress of the upper layers are only slightly indicated in the wind stress. Thus only a poor relation between wind stress and apparent stresses can be derived directly.

The main sources of errors yielding differences between the apparent and the real stresses are the computed sea level inclinations and the smoothed wind fields. Inspection of (1) and (2) shows that errors in $\nabla \zeta$ result in apparent stresses $d \tau / d z=$ const, i.e., a linear variation of $\tau$ with $z$. Instead of removing this beforehand, by which method a large part of the variations of the Ekman stress with depth could be misinterpreted, we produce an "apparent wind stress," derive the relation between apparent stress and apparent wind stress, and then separate the linear varying stresses.

A modification of the wind stress given by the Weather Service is justified because the analysis scheme of weather prediction models contains spatial and temporal filtering and 

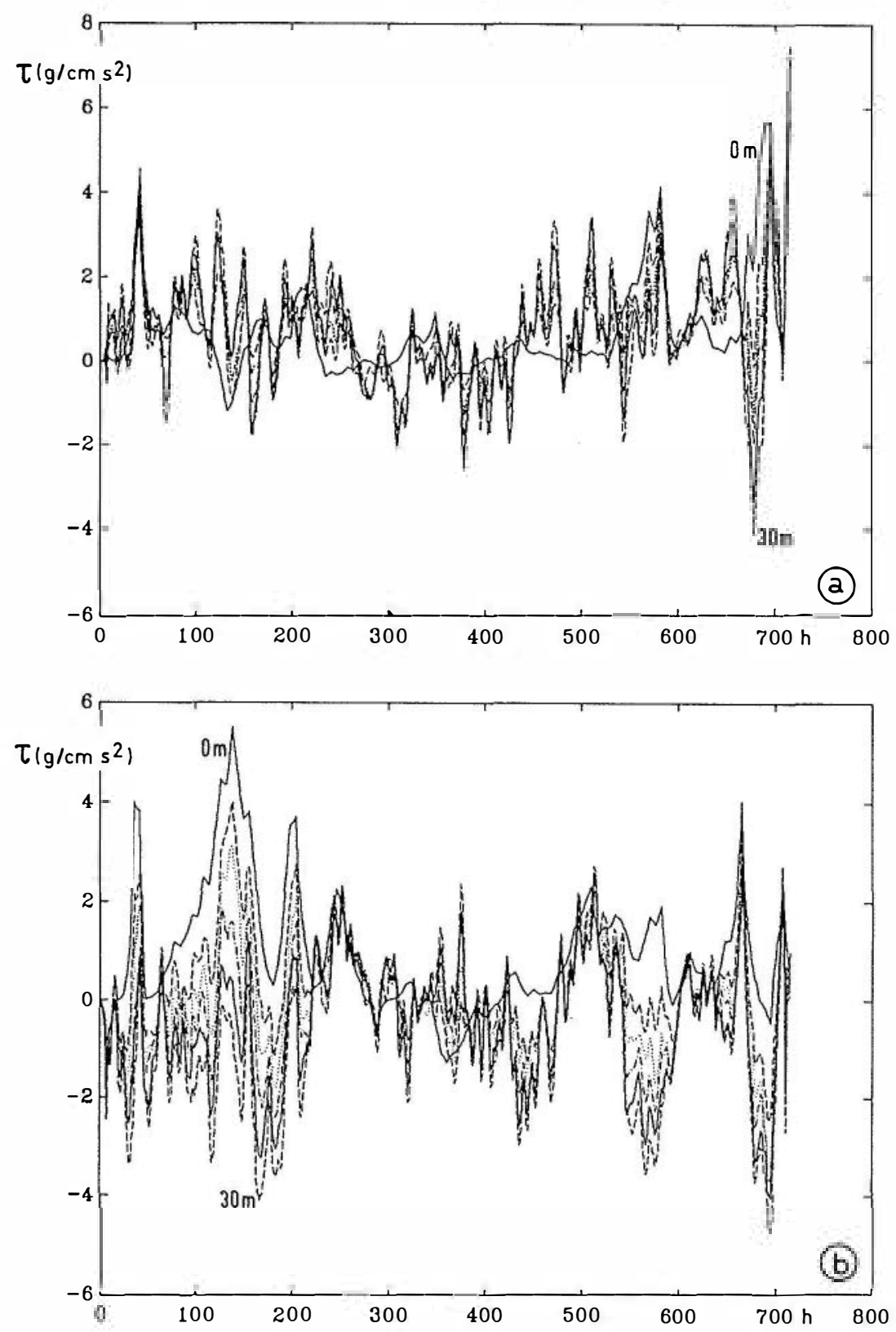

Fig. 7. Wind stress ( $\tau_{0}$, solid line) and apparent stresses at depths of $10 \mathrm{~m}$ (dashed line), $15 \mathrm{~m}$ (dotted line), $20 \mathrm{~m}$ (dashed-dotted line), $25 \mathrm{~m}$ (lower solid line), and $30 \mathrm{~m}$ (lower dashed line) as computed from (1) and (2), for $(a) x$ component and (b) $y$ component.

thus produces a smoothed time series of the wind stress. Furthermore, sampling at 6-hour intervals and spline interpolation further smoothes $\tau_{0}$.

At the sea surface the dynamic boundary condition requires the equality of wind stress and stress in the water. We assume that the fluctuations seen at 10-30 $\mathrm{m}$ transform uniformly into the surface stress; thus

$$
\tau_{10}=D_{f} \cdot \tau_{0}
$$

The tensor $D_{f}$ is determined from $\tau_{10}$ and $\tau_{0}$ by the method of least squares. Then

$$
\tau_{00}=D_{f}^{-1} \cdot \tau_{10}
$$

yields an apparent wind stress, which is used to derive relations between wind stress and stress in the water.

The apparent wind stress $\tau_{00}$ is depicted in Figure 9 together with the original data $\tau_{0}$. The general shape of the curves is the same, but $\tau_{00}$ becomes wigglier in accordance with $\tau_{10}$. The elements of $D_{f}^{-1}$ and $D_{f}$ are

$$
D_{f}^{-1}=\left(\begin{array}{cc}
0.945 & -0.244 \\
0.496 & 1.063
\end{array}\right) \quad D_{f}=\left(\begin{array}{rr}
0.945 & 0.217 \\
-0.441 & 0.840
\end{array}\right)
$$

The statistics of the two wind stresses a re approximately the same. The mean values of $\tau_{0}^{x}$ and $\tau_{0}^{y}$ are $\left\langle\tau_{0}^{x}\right\rangle=0.7$ and $\left\langle\tau_{0}^{y}\right\rangle$ $=1.0$ compared with $\left\langle\tau_{00}^{x}\right\rangle=0.7$ and $\left\langle\tau_{00}^{y}\right\rangle=0.8$. The 


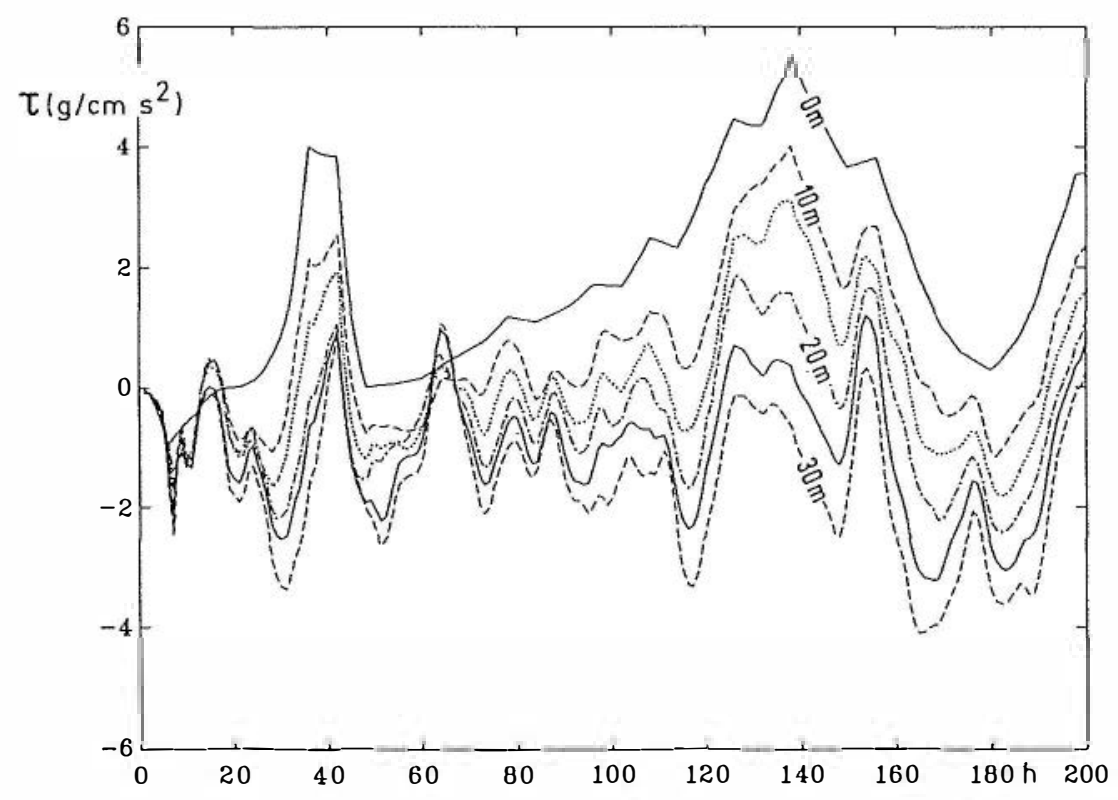

Fig. 8. Same as Figure $7 b$ for the first 200 hours on a larger scale.

corresponding values for the standard deviations are 1.2 and 1.3 for $\tau_{0}^{x}$ and $\tau_{0}^{y}$ compared with 1.0 and 1.2 for $\tau_{00}^{x}$ and $\tau_{00}^{y}$, respectively. As can be seen from the spectra (not shown) and from Figure 9, some energy is shifted toward higher frequency by this transformation, but otherwise the spectra are identical.

\section{Statistics of the Apparent Stress Field}

As was mentioned in the preceding section, the apparent stresses may contain several errors. Furthermore, contributions may not be related to the wind stress but may result from turbulence due to tidal currents, etc. To investigate whether dominant characteristics of the Ekman relations are present in the apparent stresses, we first compute simple statistics.

The Ekman spiral is generally plotted as hodograph in the velocity plane. Its vertical derivative, the stress spiral, is given by

$$
\begin{array}{r}
\tau^{x}=-\tau_{0}^{y} \sin \gamma z e^{\gamma z} \quad \tau^{y}=\tau_{0}^{y} \cos \gamma z e^{\gamma z} \\
y=(f \rho / 2 \mu)^{1 / 2}
\end{array}
$$

if $z$ is pointing upward $(-H<z<0)$ and $\tau_{0}^{x}=0$. Figure 10 depicts $\tau$ for $\gamma z=0.1 n \pi$ and a wind blowing in the $y$ direction. The Ekman depth is $\gamma D=\pi$. Contrary to the hodograph of the velocity, the stress spiral is not turned $45^{\circ}$ to the right, but points into the wind direction at the sea surface. We must have this in mind if we compare observed deflections of the stress from the direction of the wind stress.

Figure 11 shows scatter diagrams of the stress $|\tau|$ in 10-, 15-, 20-, 25-, and 30-m depths, as a function of the wind stress $\left|\tau_{00}\right|$. These diagrams clearly show that the stress at all depths is linearly related to the wind stress. The scatter increases with depth. Two aspects may be noteworthy: (1) Ekman's theory yields $|\tau|=\left|\tau_{0}\right| \exp (\gamma z)$ according to (6). From Figure 11 we conclude that the inclination of the regression lines decrease with depth, but not as is to be expected from the Ekman relation with constant $\mu$. Evaluating $\exp \left(\gamma z_{i}\right)$ results in $\mu=553,2214,1143,1008$, and 1540 cgs units for $10,15,20,25$, and $30 \mathrm{~m}$, respectively. (2) The intercept of the regression line at $\left|\tau_{00}\right|=0$ increases with depth. That a wind-induced stress does not vanish when the wind stress becomes zero is to be expected, because the time scales of the wind field are different from those of the ocean. The ocean will remain turbulent until the turbulence decays. However, whether or not the increase of $|\tau|$ with depth for low wind velocities is real will be discussed in the next sections.

In Figure 12 we display histograms of the angle of deflection for the same depths. The angle given in the top right corner of each plot is the median value. Positive angles mark deflections to the right of the wind. As can be seen from these figures the deflection of the stress to the right relative to the instantaneous wind stress increases from $21.1^{\circ}$ in $10-m$ depth to $46.2^{\circ}$ in $30-\mathrm{m}$ depth. The histograms broaden with increasing depth, but the deflections are a strong indication that some type of Ekman spiral dominates within the upper $30 \mathrm{~m}$. Note that this deflection is not apparent in the trajectories (Figure 5) and that these angles of deflection are based on the apparent stresses, which will be further decomposed in the following sections. They do not characterize the pure Ekman spiral.

\section{Separation of the Ekman Stresses}

Ekman's theory states that the stress (and therefore also the currents) within the Ekman layer can be derived directly from the wind stress. The Ekman solution can be written as

$\tau(z)=D(z) \cdot \tau_{0} \quad D(z)=e^{\gamma z}\left(\begin{array}{rr}\cos \gamma z & -\sin \gamma z \\ \sin \gamma z & \cos \gamma z\end{array}\right)$

with $(-\infty<z \leq 0)$. 

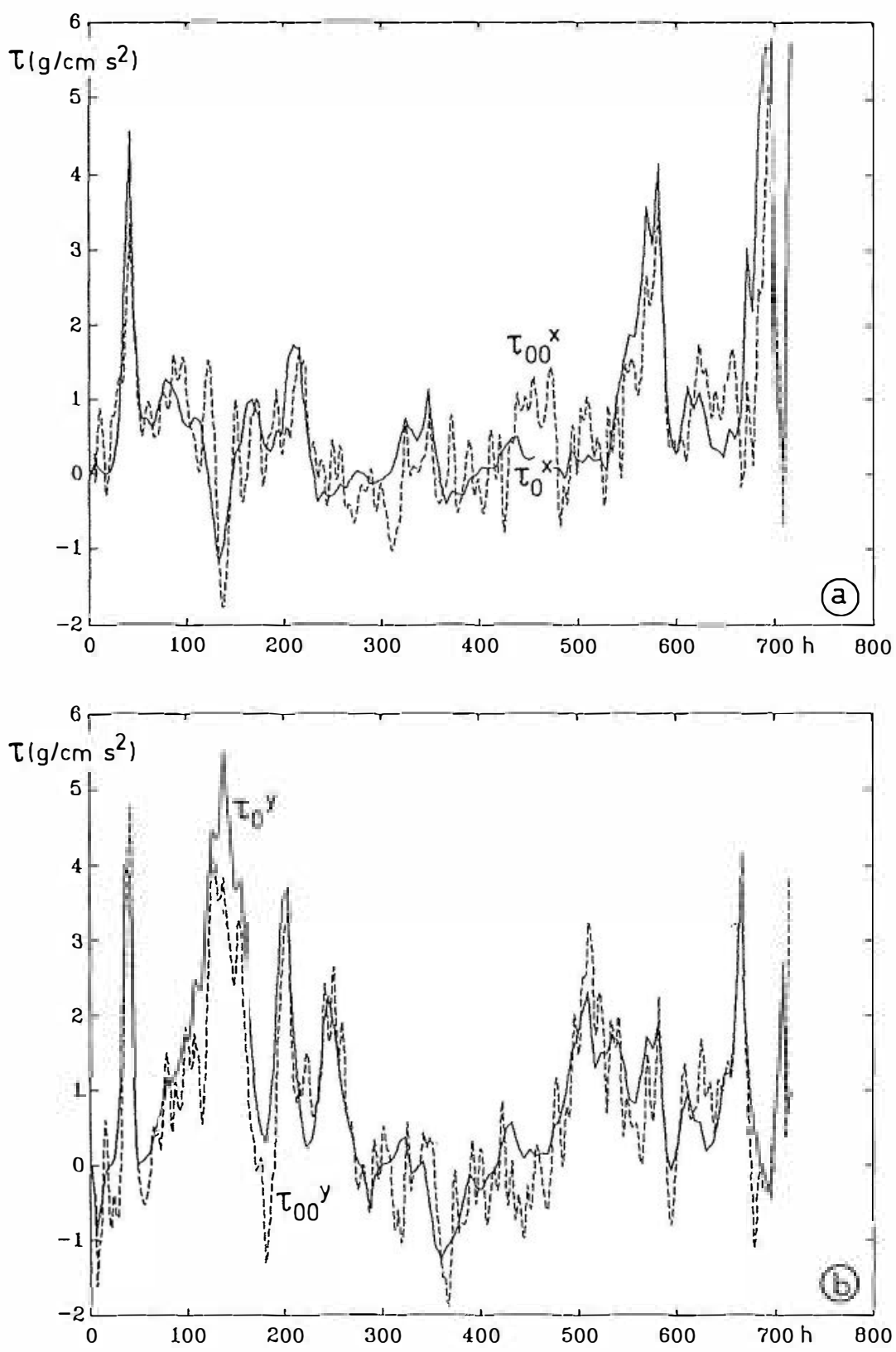

Fig. 9. Wind stress $\tau_{0}$ (solid line) and apparent wind stress $\tau_{00}$ (dashed line) according to equation (4), for $(a)$ east component and $(b)$ north component.

The tensor $D(z)$ describes the rotation and the exponential decrease of the stress $\tau$ with depth. The sine and cosine functions as well as the exponential decay are a consequence of $\mu=$ const, yielding $\gamma=$ const.

Similarly, the unsteady Fredholm solution can be brought into the equivalent form

$$
\tau(z)=-\frac{z}{2(\pi \nu)^{1 / 2}} \int_{0}^{t} D\left(z, t^{\prime}\right) \cdot \frac{\tau_{0}\left(t-t^{\prime}\right)}{\left(t^{\prime 3}\right)^{1 / 2}} d t^{\prime} \quad \nu=\frac{\mu}{\rho}
$$

where the tensor is given by

$$
D\left(z, t^{\prime}\right)=e^{-z^{2} / 4 \nu t^{\prime}}\left(\begin{array}{cc}
\cos f t^{\prime} & \sin f t^{\prime} \\
-\sin f t^{\prime} & \cos f t^{\prime}
\end{array}\right)
$$

In this case the decay of $\tau$ with depth appears more complicated and is time dependent. Averaged over an inertial period, however, the Ekman spiral emerges, and only a few hours of the history of the wind stress modify the results described by (7). We want to proof whether or not a generalized version of (7) holds, i.e., whether part of the apparent stress field can be related to a tensor $D_{W}$ under all weather conditions directly to the wind stress $\tau_{00}$, without imposing the concept of constant eddy viscosity. Thus we expect

$$
\tau(z) \sim D_{w} \cdot \tau_{00}
$$

to hold for the entire time series and compute the components of $D_{w}, d_{w i k}$, by the method of least squares 


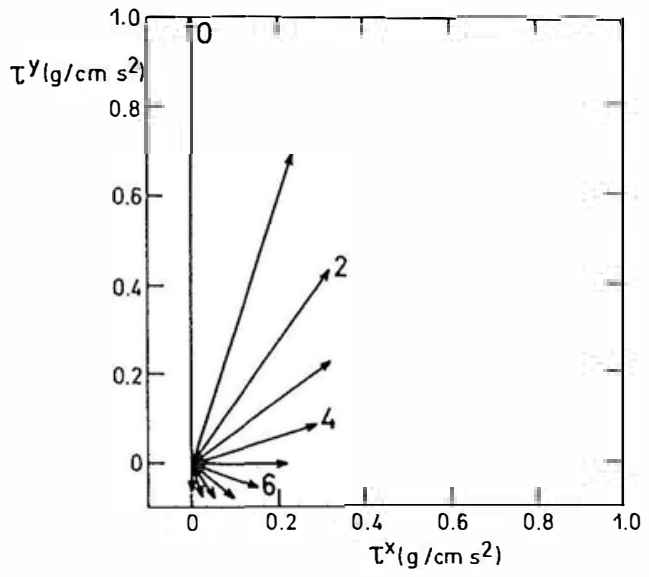

Fig. 10. Stress spiral with $\tau_{0}^{x}=0, \tau_{0}^{\gamma}=1$. The arrows are plotted for $\gamma z=0.1 n \pi, n=0, \cdots, 10$.

$$
\begin{aligned}
& \sum_{i}\left[\tau^{x}(z)-d_{W 11} \tau_{00}^{x}-d_{W 12} \tau_{00}^{y}\right]^{2}=\text { Min } \\
& \sum_{i}\left[\tau^{y}(z)-d_{W 21} \tau_{00}^{x}-d_{W 22} \tau_{00}^{y}\right]^{2}=\operatorname{Min}
\end{aligned}
$$

separately for each level $z$.

Equations (11) are solved independently for the elements $d_{W 11}, d_{W 12}$, and $d_{w_{21}}, d_{W 22}$, respectively. They are listed in Table 1. The diagonal elements $d_{W_{11}}$ and $d_{W 22}$ mainly determine the decay with depth, the off-diagonal elements are important for the rotation with depth.

We define

$$
\tau_{W}(z)=D_{W} \cdot \tau_{00}
$$

as wind-induced stress, i.e., $\tau_{w}(z)$, is the part of the apparent stress $\tau(z)$ that can be related to the wind stress by means of $D_{W}$ and is determined by (12). It is plotted in Figure 13 together with the apparent stress $\tau(z)$. At $10-\mathrm{m}$ depth the entire apparent stress is wind induced as a consequence of the transformation (4), i.e., $D_{W}(10 \mathrm{~m})=D_{f}$. With increasing depth the part of the stress that can be related to the wind stress becomes smaller. Note that the agreement between both stresses $\tau_{w}$ and $\tau$ is nearly independent from the weather conditions, i.e., relation (12) holds for all wind conditions observed during this 4-week experiment, regardless of whether the wind is weak or strong.

The tensor $D_{W}$ that describes the transformation of $\tau_{00}$ into $\tau_{W}$ will now be decomposed into an Ekman-type tensor $D_{E}$ and the remaining residuum $D_{R}$. The Ekman tensor requires $d_{E 11}=d_{E 22}$ and $d_{E 21}=-d_{E 12}$. Table 1 suggests defining $D_{E}$ by the averages of the elements,

$$
\begin{array}{cc}
d_{E 11}=\frac{d_{W 11}+d_{W 22}}{2} & d_{E 22}=d_{E 11} \\
d_{E 12}=\frac{\left|d_{W 12}\right|+\left|d_{W 21}\right|}{2} & d_{E 21}=-d_{E 12}
\end{array}
$$

The residuum tensor is given by $D_{R}=D_{W}-D_{E}$ and is characterized by $d_{R 22}=-d_{R 11}, d_{R 21}=d_{R 12}$. The principal elements of both tensors are listed in Table 2. By definition,
$D_{W}=D_{E}+D_{R}$ exactly. Their depth distribution can be described by

$$
\begin{aligned}
& d_{E 11}=1.0-0.076 \times 10^{-1} n \\
& \quad-2.804 \times 10^{-2} n^{2}+2.628 \times 10^{-3} n^{3} \\
& d_{E 12}=2.177 \times 10^{-1} n-2.861 \times 10^{-2} n^{2} \\
& \quad+1.893 \times 10^{-3} n^{3} \\
& d_{E 21}=-d_{E 12} \quad d_{E 22}=d_{E 11} \quad \\
& d_{R 11}=3.308 \times 10^{-2} n \quad d_{R 22}=-d_{R 11} \\
& d_{R 12}=-4.073 \times 10^{-2} n \quad d_{R 21}=d_{R 12}
\end{aligned}
$$

where $z=n d z, d z=5 \mathrm{~m}$, and $z$ is positive downward in this approximation $(n=0, \cdots, 6)$.

In Figure 14 we depict the tensor elements of $D_{E}$ and $D_{R}$, which determine the Ekman stress $\tau_{E}$ and the residuum stress $\tau_{R}$ according to

$$
\begin{aligned}
\tau_{E} & =D_{E} \cdot \tau_{00} \\
\tau_{R} & =D_{R} \cdot \tau_{00}
\end{aligned}
$$

The values at $z=0$ are added and reflect the dynamic boundary condition.

From the first equation (15) we can plot the Ekman stress spiral (as usual for $\tau_{00}^{x}=0, \tau_{00}^{y}=1$ ); the Ekman velocity spiral is then given by

$$
u=\frac{1}{f} \frac{d\left(d_{E 11}\right)}{d z} \quad v=-\frac{1}{f} \frac{d\left(d_{E 12}\right)}{d z}
$$

Both spirals are shown in Figure 15, using the relations (14). The Ekman tensor rotates the stress vector uniformly from the wind stress direction to a direction $53^{\circ}$ toward the right $(5$ $\mathrm{m}, 11^{\circ} ; 10 \mathrm{~m}, 21^{\circ} ; 15 \mathrm{~m}, 29^{\circ} ; 20 \mathrm{~m}, 38^{\circ} ; 25 \mathrm{~m}, 46^{\circ} ; 30 \mathrm{~m}, 53^{\circ}$ ). The angle of deflection is given by $\tan \alpha=d_{E 12} / d_{E 22}$ and is inconsistent with a constant $\mu$. The magnitude of the stress does not decrease exponentially but according to $\left(d_{E 11}^{2}+\right.$ $\left.d_{E 12}^{2}\right)^{1 / 2}$.

The Ekman velocity spiral (Figure $15 b$ ) shows a deflection of $10^{\circ}$ to right of the wind direction at $2.5 \mathrm{~m}, 27^{\circ}$ at $7.5 \mathrm{~m}, 42^{\circ}$ at $12.5 \mathrm{~m}, 50^{\circ}$ at $17.5 \mathrm{~m}, 52^{\circ}$ at $22.5 \mathrm{~m}$, and $45^{\circ}$ at $27.5 \mathrm{~m}$ according to (14). The deflection to the right is determined by the ratio $u / v$. As the diagonal element $d_{E 11}$ varies less with depth in the upper layers than the off-diagonal element $d_{E 12}$ (Figure 14), the deflection is less than $45^{\circ}$. Thus we conclude that the simple concept of constant eddy viscosity for a homogeneous water mass, which results in (7), does not fit the data, whereas the tensor $D_{E}+D_{R}$ gives a good approximation to the data. It remains to interpret the residuum stress $\tau_{R}$, the second part of the wind-induced stress. It is negligible within the upper $10 \mathrm{~m}$ but gains on relative importance with depth owing to its linear increase.

As was stated already in section 3, a linear increasing stress means constant $d \tau / d z$ and thus produces a barotropic current like $g \nabla \zeta$ according to (1) and (2). The increase of $D_{R}$ of 0.2 over $30-\mathrm{m}$ depth corresponds to an inclination of the sea surface of $0.9 \mathrm{~cm}$ over $100 \mathrm{~km}$, or about $5 \mathrm{~cm}$ across the North Sea. This seems to be within the error bars of the barotropic model, from which $\zeta_{x}$ and $\zeta_{y}$ have been deter- 

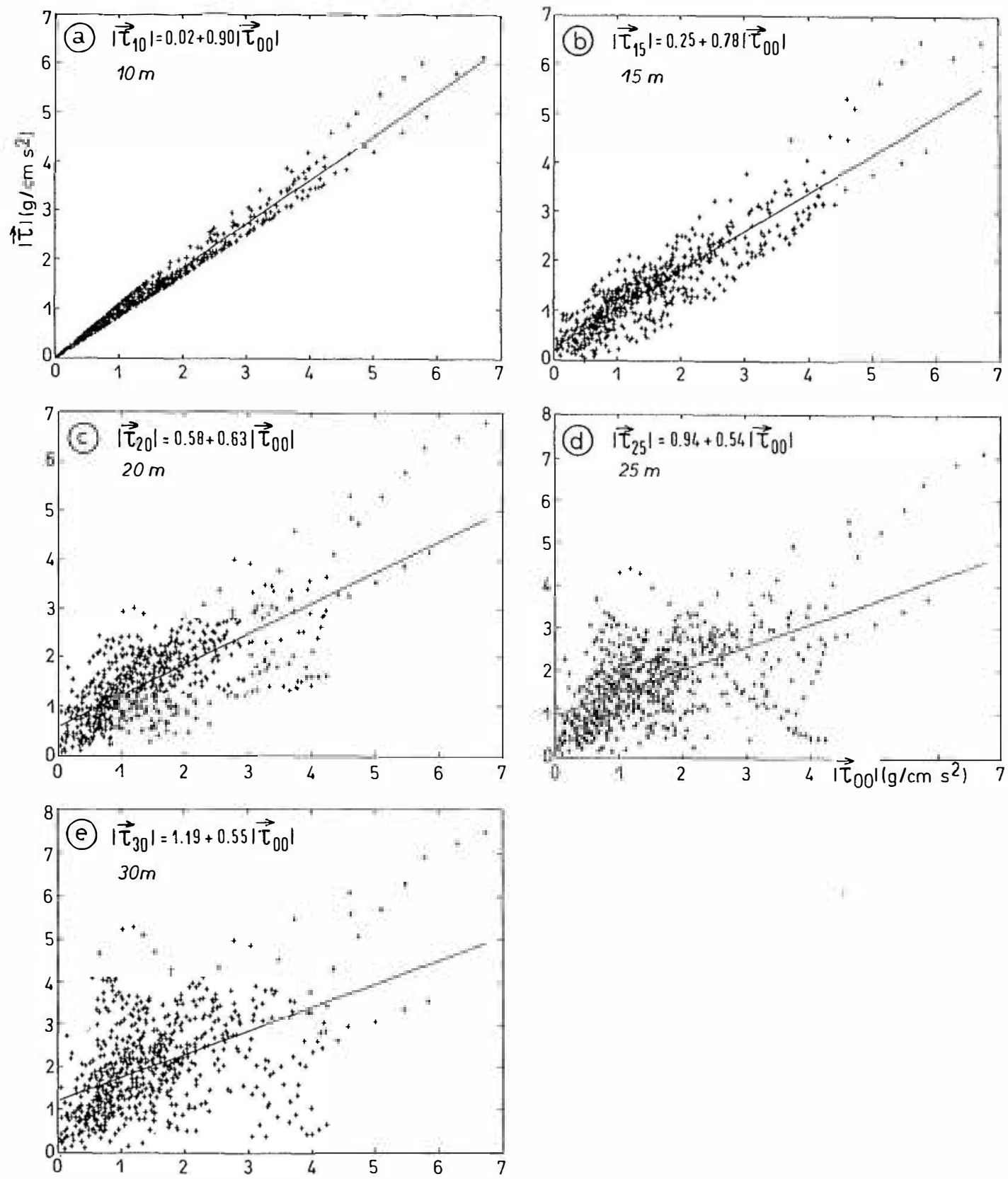

Fig. 11. Stress $|\tau|$ at depths of (a) $10 \mathrm{~m},(b) 15 \mathrm{~m},(c) 20 \mathrm{~m},(d) 25 \mathrm{~m}$, and (e) $30 \mathrm{~m}$ as a function of wind stress $\left|\boldsymbol{\tau}_{00}\right|$. The solid line is the linear regression.

mined. We therefore interpret the apparent linear increasing stress $\tau_{R}$ as a correction to the computed sea level inclinations $\zeta_{x}, \zeta_{y}$, and not as real stress. The only wind-induced stress which remains is the Ekman stress $\tau_{E}$.

\section{The Viscosity Tensor}

Using $\tau_{E}$ in (1) and (2) as stress and setting $\zeta_{x}, \zeta_{y}$ equal to zero we can compute the pure Ekman currents $u_{E}, v_{E}$ for each level. There is no need to introduce an eddy viscosity into the equations of motion; they are solved much easier with the stresses as driving forces, given by the wind stress via $D_{E}$.

However, to complete the analysis, we investigate whether a meaningful eddy viscosity is consistent with the data. We first test whether or not a scalar eddy viscosity is a useful quantity and whether or not it is related to the wind stress as has been proposed by several authors (see section 1).

The computed Ekman velocities due to $\tau_{E}$ and the wind stress $\tau_{00}$ are shown in Figure 16 for the $u$ and $v$ components. They are obtained by numerically integrating (1) and (2) with $\zeta=0$. They vary little with depth. The mean values of the speed are 5.2, 4.3, 4.0,3.7, and $3.1 \mathrm{~cm} \mathrm{~s}^{-1}$ at 7.5-, 12.5-, 17.5-, 22.5-, and 27.5-m depth, respectively. This indicates again that $\mu$, if meaningful at all, must be large Eddy viscosity and stress are usually related according to

$$
\mu\left(\partial u_{E} / \partial z\right)=\tau_{E}^{x} \quad \mu\left(\partial v_{E} / \partial z\right)=\tau_{E}^{y}
$$

Knowing the relation between wind stress and the stress in the interior (equation (14)), we could now use the ob- 

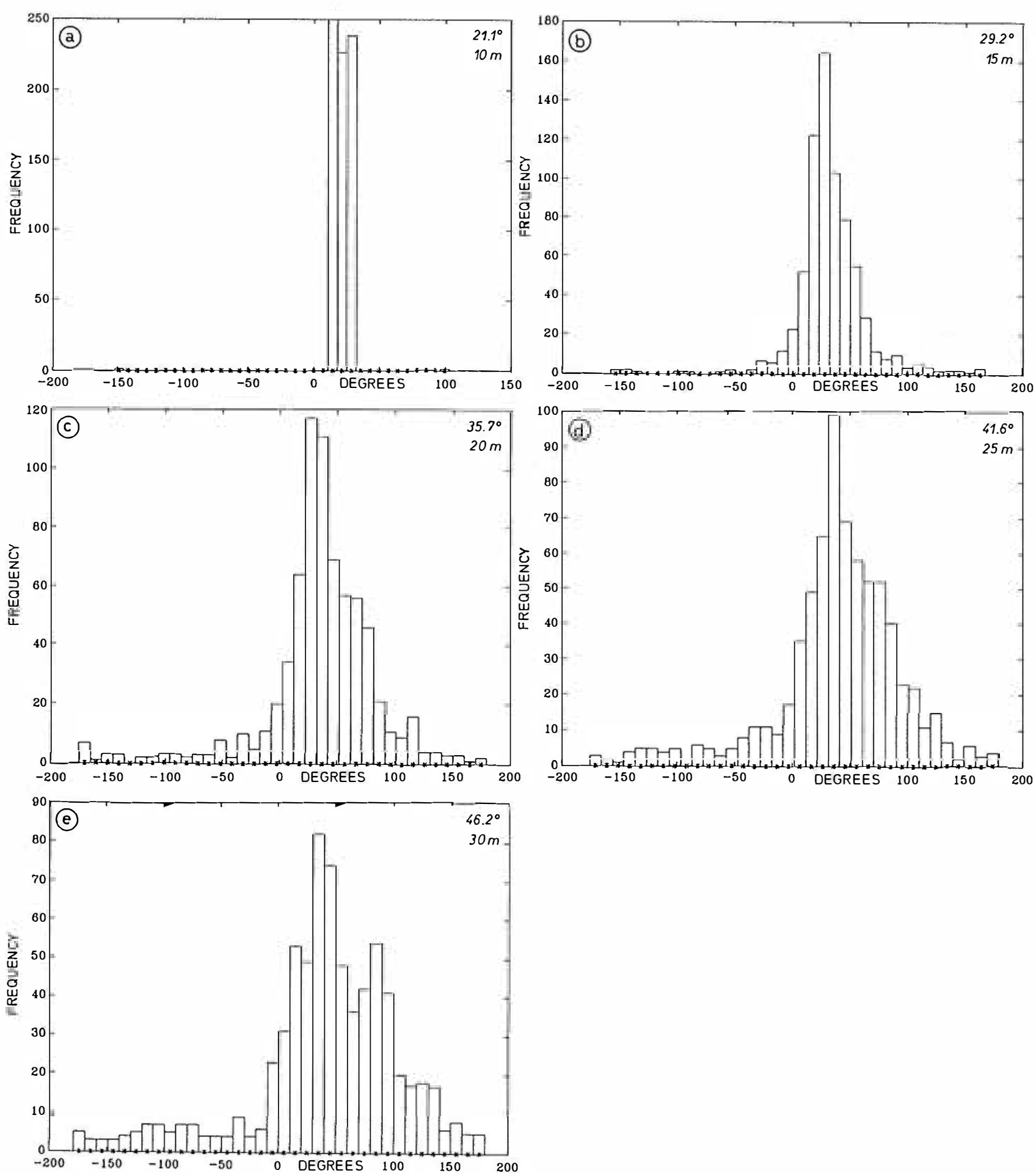

Fig. 12. Histograms of the angle of deflection between the apparent stress $|\tau|$ at depths of (a) $10 \mathrm{~m},(b) 15 \mathrm{~m},(c) 20$ $\mathrm{m},(d) 25 \mathrm{~m}$, and (e) $30 \mathrm{~m}$ and the wind stress $\left|\tau_{00}\right|$. Positive angles are deflections to the right of the wind stress.

served wind stress $\tau_{0}$ for the simulation of the Ekman velocities. However, the use of $\tau_{0}$ or $\tau_{00}$ in these simulations gives only slight differences, as is to be expected from Figure 9. We therefore use $\tau_{00}$ also through the remaining computations.

The computation of $\mu$ from data is very sensitive, because the (very small) changes of $\mathbf{v}_{E}$ with depth enter the analysis.
Squaring equations (23) and adding, we obtain with $\tau_{E}=$ $D_{E} \cdot \tau_{00}$

$\mu=\left(d_{E 11}^{2}+d_{E 12}^{2}\right)^{1 / 2} /\left[\left(\partial u_{E} / \partial z\right)^{2}+\left(\partial v_{E} / \partial z\right)^{2}\right]^{1 / 2}\left|\tau_{00}\right|$

This gives apparently a relation between $\mu$ and the magnitude of the wind stress, $\left|\tau_{00}\right|$. However, evaluation of (18), as 
TABLE 1. Elements $d_{w i k}$ of the Tensor $D_{w}$ That Relates the Apparent Stresses to the Wind Stress $\tau_{00}$

\begin{tabular}{ccccc}
\hline Depth, m & $d_{w 11}$ & $d_{w 12}$ & $d_{w 21}$ & $d_{w 22}$ \\
\hline 10 & 0.9445 & 0.2167 & -0.4407 & 0.8397 \\
15 & 0.8900 & 0.3080 & -0.6027 & 0.7132 \\
20 & 0.8132 & 0.3674 & -0.6965 & 0.5492 \\
25 & 0.7688 & 0.4040 & -0.8062 & 0.4208 \\
30 & 0.7130 & 0.4638 & -0.9090 & 0.3101
\end{tabular}

depicted in Figure 17, shows that the factor of $\left|\tau_{00}\right|$ nearly completely cancels this linear relationship. Formally, we obtain $\mu=1155+26\left|\tau_{00}\right|$ at 10 -m depth, but the large scatter allows us to state that $\mu$ is not dependent on the wind stress;
TABLE 2. Elements $d_{E i k}$ and $d_{R i k}$ of the Ekman Tensor $D_{E}$ and the Residuum Tensor $D_{R}$

\begin{tabular}{ccccc}
\hline Depth, $\mathrm{m}$ & $d_{E 11}$ & $d_{E 12}$ & $d_{R 11}$ & $d_{R 12}$ \\
\hline 10 & 0.8921 & 0.3287 & 0.0524 & -0.1120 \\
15 & 0.8016 & 0.4554 & 0.0884 & -0.1474 \\
20 & 0.6812 & 0.5320 & 0.1320 & -0.1646 \\
25 & 0.5948 & 0.6051 & 0.1740 & -0.2011 \\
30 & 0.5115 & 0.6864 & 0.2015 & -0.2226
\end{tabular}

its level remains constant whether it is stormy or not. We therefore reject any of the proposed relations between $\mu$ and the wind speed mentioned in section 1. In evaluating (18) the values of $\partial v_{E} / \partial z$ have been limited to the mean value plus
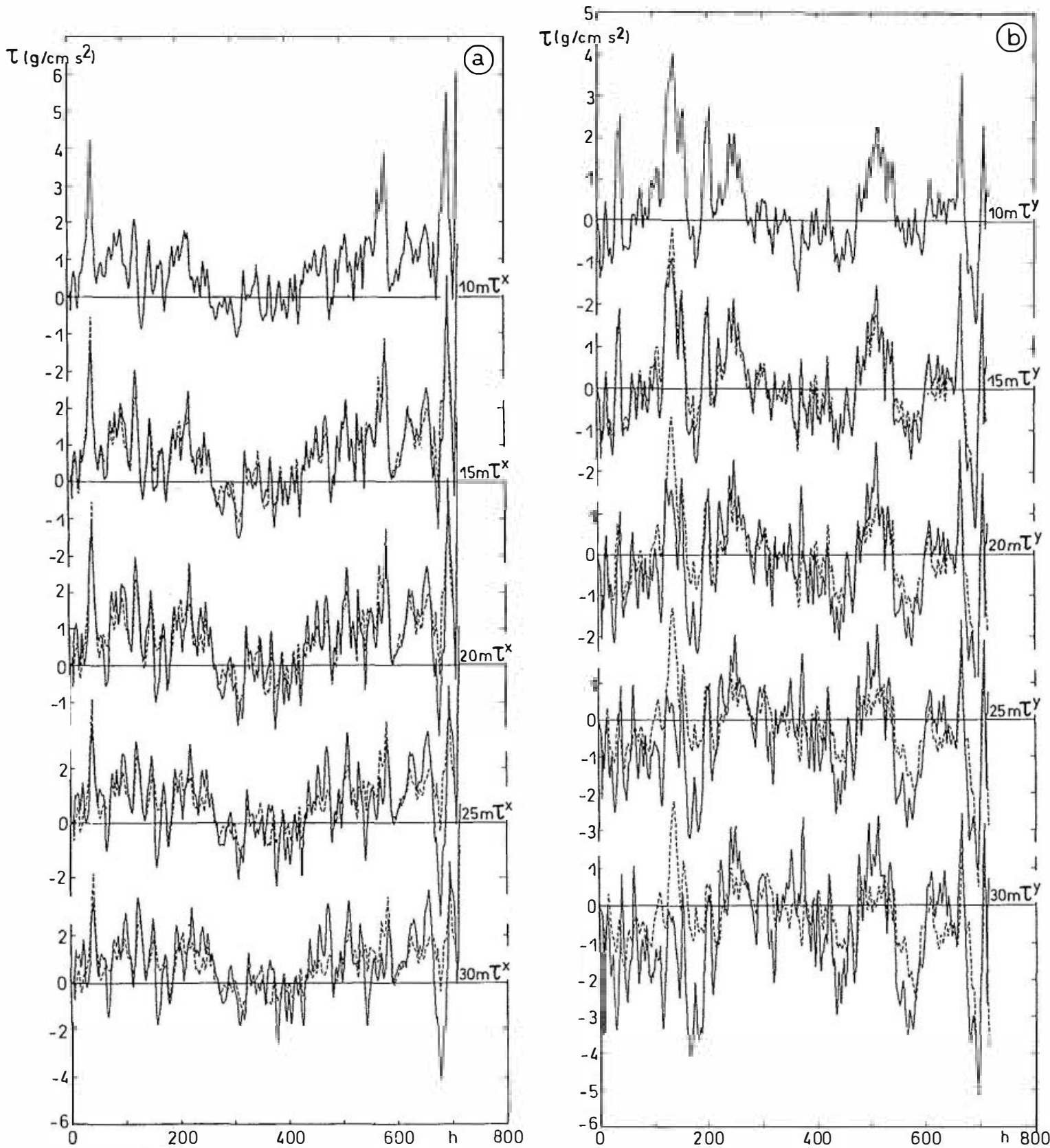

Fig. 13. Apparent stress $\tau$ (solid lines) and wind-induced stress $\tau_{1 W}$ (dashed lines) in 10-, 15-, 20-, 25-, and 30- $\mathrm{m}$ depth. for $(a) x$ component and (b) y'component. 

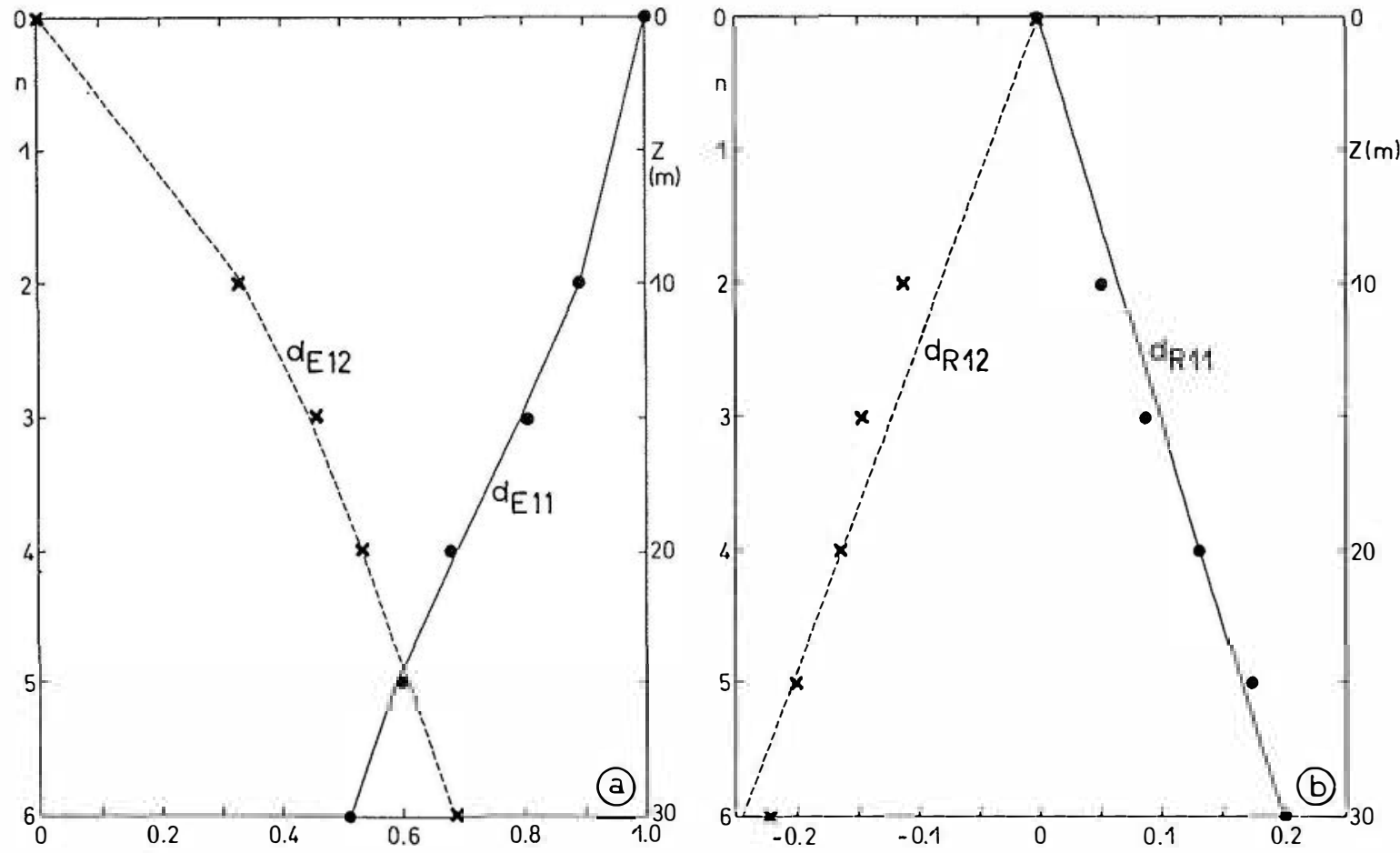

Fig. 14. Elements of $(a)$ the Ekman tensor $D_{E}$ and $(b)$ the residuum tensor $D_{R} ; z=n d z, d z=5 \mathrm{~m}$. Circles and crosses are the values in Table 2.

three times the standard deviations. The large scatter is due to small velocity shears. Similar results are obtained for the other levels.

The data further show that a scalar eddy viscosity is not a meaningful quantity for describing the stress. We demonstrate this by plotting the velocity differences of the Ekman currents $\mathbf{v}_{E}$ between $10-\mathrm{m}$ and $30-\mathrm{m}$ depth together with the stress averaged over the $15-\mathrm{m}, 20-\mathrm{m}$, and $25-\mathrm{m}$ depth layers, which is an approximation to (17). This is shown in Figure
18. Obviously, there is no possibility to transform $\Delta u$ and $\Delta v$ (solid lines) into $\left\langle\tau^{x}\right\rangle,\left\langle\tau^{y}\right\rangle$ (dashed lines), by a simple multiplication with a scalar quantity $\mu$, as proposed by (17).

Ertel [1937] first proposed relating the Reynolds stress tensor to the mean velocity by a tensor relation. However, his formulation is not useful in this context, because it is not the three-dimensional structure of turbulence which is important in the mixed layer but the relation between stress and vertical shear of the mean velocity. We put
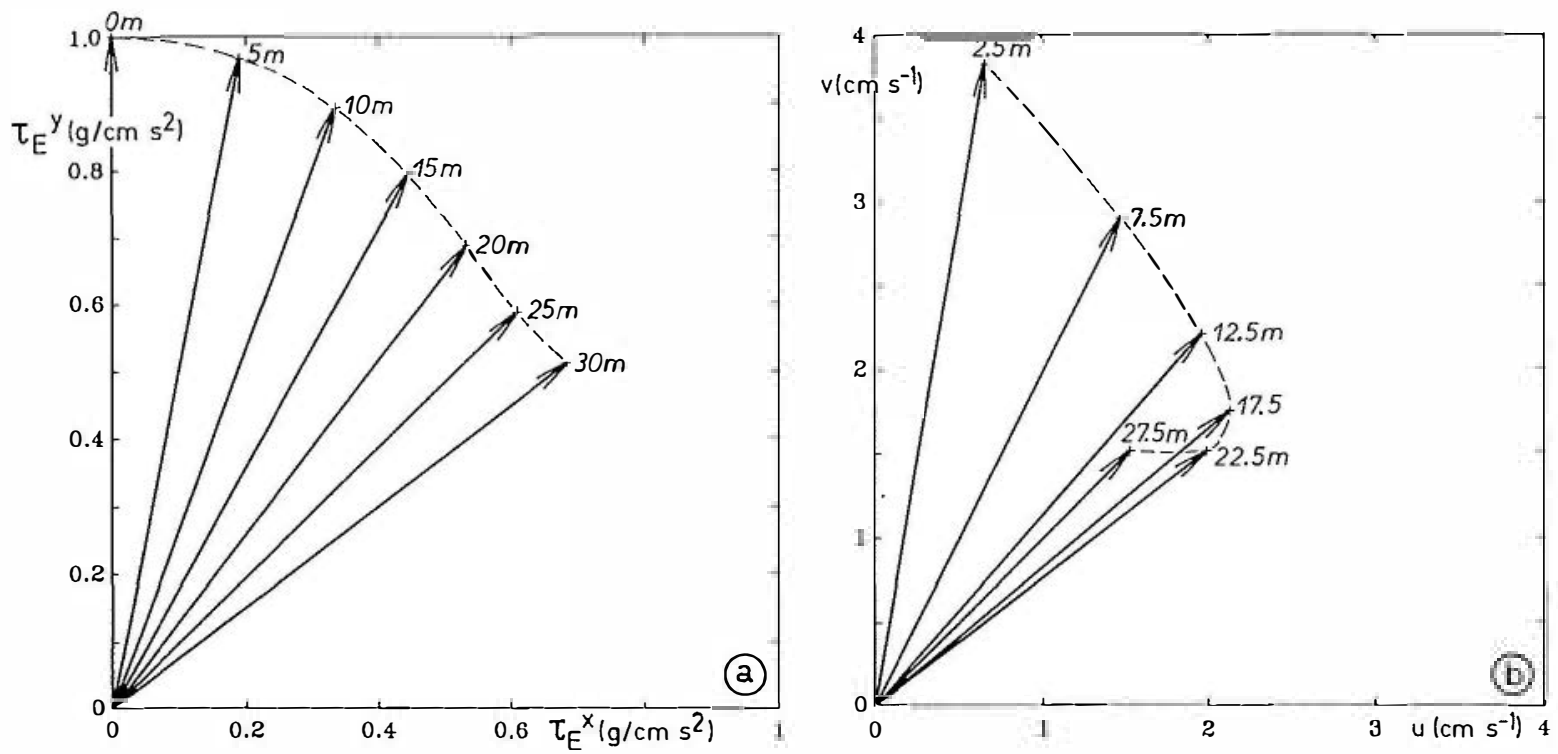

Fig. 15. (a) Ekman stress spiral and (b) Ekman velocity spiral based on (14) for $\tau_{00}^{x}=0, \tau_{0}^{\gamma_{0}}=1$. 

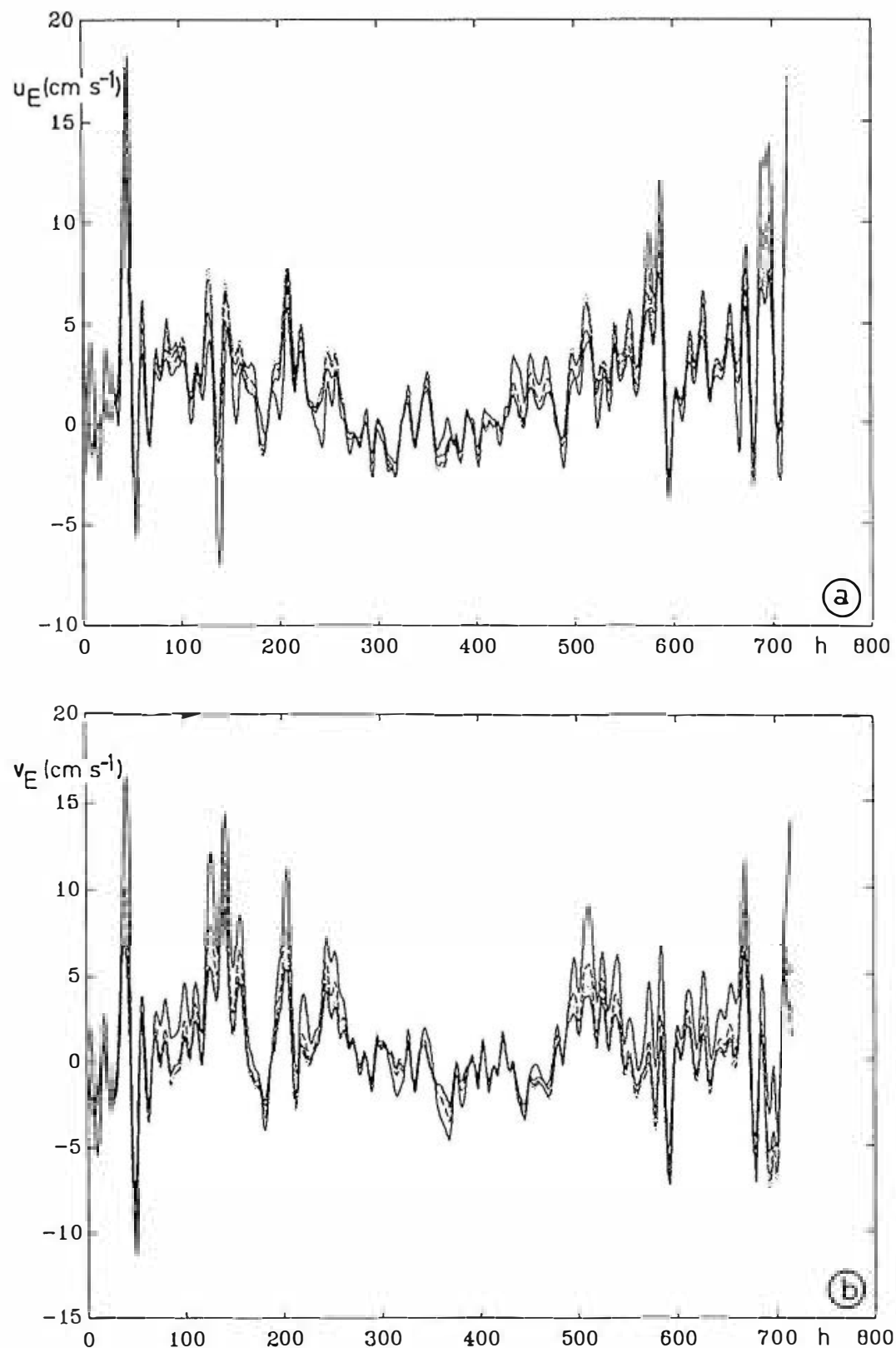

Fig. 16. Ekman velocities $(a) u_{E}$ and $(b) v_{E}$ in $7.5-\mathrm{m}, 12.5-\mathrm{m}, 17.5-\mathrm{m}, 22.5-\mathrm{m}$, and $27.5-\mathrm{m}$ depth due to the Ekman stress $\tau_{E}$ and the surface stress $\tau_{00}$. Line types as in Figure 6.

$$
\tau_{E}=A \cdot \frac{\partial \mathbf{v}_{E}}{\partial z}
$$

where $A$ will be called eddy viscosity tensor. Its elements $a_{11}, a_{22}, a_{12}$, and $a_{21}$ are again determined from $\tau_{E}$ and $\mathbf{v}_{E}$ by the method of least squares. The result is tabulated in Table 3 and plotted in Figure 19a. The tensor elements fit remarkably well a relationship $a_{11}=a_{22}, a_{21}=-a_{12}$. In Figure 20 we show the components of $\tau_{E}$ according to (14) and (15) and $A \cdot \partial \mathbf{v}_{E} / \partial z$ according to (19) using the values in Table 3. The eddy viscosity tensor not only is able to simulate the major trends in the records but also describes many details of the fluctuations. The fit appears equally good under storm conditions and in calm weather.

An outstanding feature in the vertical distribution of the eddy viscosity tensor (Figure 19a) is its extreme values in 15- to 20-m depth and the rapid decay toward both the surface and greater depth. A possible interpretation of this result is that the major contributions to the eddy viscosity tensor stem from the velocities in the Langmuir circulation cells. Weller et al. [1985] and Smith et al. [1987] have shown that intense downwind currents associated with vertical velocities in the range of $15-30 \mathrm{~cm} \mathrm{~s}^{-1}$ in $15 \mathrm{~m} \mathrm{~s}^{-1}$ winds occur in Langmuir cells. They appear as events, and the largest ones are observed consistently in the depth range $10-30 \mathrm{~m}$ below the surface. They may be responsible for the maximum in $\left\langle u^{\prime} w^{\prime}\right\rangle$ and thus in the viscosity tensor.

The possibility of describing the Ekman stresses by an eddy viscosity tensor is not surprising. The eddy viscosity tensor can be directly derived from the Ekman tensor. As the latter holds under all wind conditions during our obser- 


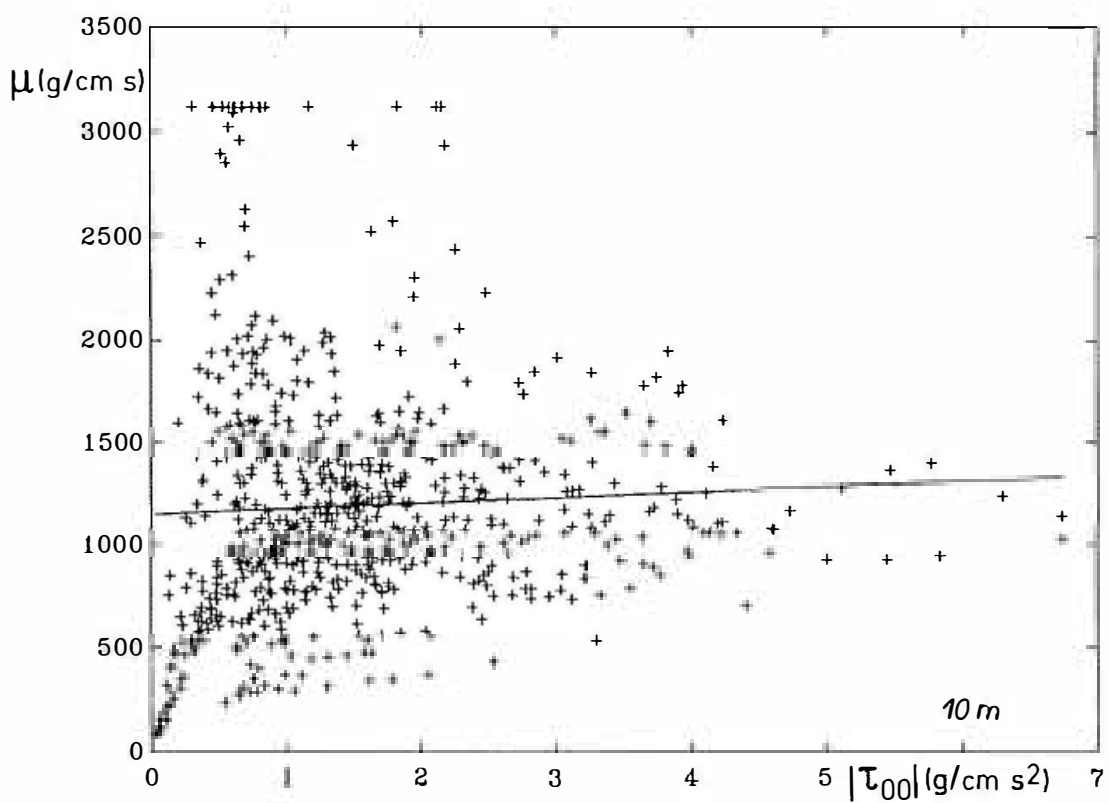

Fig. 17. Scatterplot of $\mu$ as function of $\left|\tau_{(x)}\right|$ at $10-\mathrm{m}$ depth according to (18). The solid line is the regression line.

vational period, we choose $\tau^{x}=0, \tau^{y}=1$. It then follows from (15) and (19) and from the results depicted in Figure $19 a\left(a_{22}=a_{11}, a_{21}=-a_{12}\right)$ that

$\tau_{E}^{x}=d_{E 12}$

$\tau_{E}^{x}=a_{11} \frac{d u}{d z}+a_{12} \frac{d v}{d z} \quad \tau_{E}^{y}=-a_{12} \frac{d u}{d z}+a_{11} \frac{d v}{d z}$

which yields

$$
\begin{aligned}
& a_{11}=\frac{(d v / d z) d_{E 11}+(d u / d z) d_{E 12}}{(d u / d z)^{2}+(d v / d z)^{2}} \\
& a_{12}=\frac{(d v / d z) d_{E 12}-(d u / d z) d_{E 11}}{(d u / d z)^{2}+(d v / d z)^{2}}
\end{aligned}
$$

Figure $19 b$ depicts the elements $a_{11}, a_{12}$, of the eddy viscosity tensor $A$ according to these relations, where $d u / d z$ and $d v / d z$ are given by (16). The deviations in Figure $19 a$ from Figure $19 b$ are due to the numerical approximation of the velocity shears over 5-m intervals. Using (21), the stresses shown in Figure 20 are modeled exactly.

Whereas the Ekman tensor has a clear physical meaning (it describes decay and rotation of the Ekman stress from the sea surface downward) the eddy viscosity tensor according to (21) appears as a more complicated function which requires further research.

At present we wish to make only three comments:

1. If we introduce the curl of the Ekman currents and combine the two principal components of the eddy viscosity tensor to a vector,

$$
\nabla \times \mathbf{v}=-\frac{d v}{d z} \mathbf{i}+\frac{d u}{d z} \mathbf{j} \quad \mathbf{a}=\left(\begin{array}{l}
a_{11} \\
a_{12}
\end{array}\right)
$$

(21) may be written as

$$
\mathbf{a}=\frac{\left(D_{E}-\operatorname{tr} D_{E} I\right) \cdot \nabla \times \mathbf{v}}{\text { Diss }}
$$

where $\operatorname{tr} D_{E}=D_{E 11}+D_{E 22}$ is the trace of the Ekman tensor, $I$ is the unit tensor, Diss is the dissipation $(d u / d z)^{2}+$ $(d v / d z)^{2}$, and $\mathbf{i}, \mathbf{j}$, and $\mathbf{k}$ are unit vectors in the $x, y$, and $z$ directions, respectively. Thus the tensor $D_{E}-\operatorname{tr} D_{E} I$ transforms the vorticity of the Ekman currents into the tensor elements $a_{11}, a_{12}$, as the Ekman tensor $D_{E}$ transforms the wind stress into the Ekman stress.

2. The tensor elements $a_{11}, a_{12}$, play different roles in the energy transfer. The energy equation for the Ekman dynamics results from multiplying

$$
\begin{aligned}
\frac{\partial \mathbf{v}}{\partial t}+\mathbf{f} \times \mathbf{v}=\frac{1}{\rho_{0}} \frac{\partial}{\partial z}\left(A \cdot \frac{\partial \mathbf{v}}{\partial z}\right)= & \frac{1}{\rho_{0}}\left\{\frac{\partial}{\partial z}\left(a_{11} \frac{\partial \mathbf{v}}{\partial z}\right)\right. \\
& \left.+\frac{\partial}{\partial z}\left(a_{12} \frac{\partial \mathbf{v}}{\partial z}\right) \times \mathbf{k}\right\}
\end{aligned}
$$

with v. Using

$$
\begin{gathered}
\frac{\partial}{\partial z}\left(a_{11} \frac{\partial \mathbf{v}}{\partial z}\right) \cdot \mathbf{v}=\frac{\partial}{\partial z}\left(a_{11} \frac{\partial}{\partial z} \frac{\mathbf{v} \cdot \mathbf{v}}{2}\right)-a_{11} \frac{\partial \mathbf{v}}{\partial z} \cdot \frac{\partial \mathbf{v}}{\partial z} \\
\left(\frac{\partial \mathbf{v}}{\partial z} \times \mathbf{k}\right) \cdot \mathbf{v}=-\left(\frac{\partial \mathbf{v}}{\partial z} \times \mathbf{v}\right) \cdot \mathbf{k}
\end{gathered}
$$

we arrive at

$$
\begin{aligned}
\frac{\partial}{\partial t} \frac{\mathbf{v} \cdot \mathbf{v}}{2}=\frac{\partial}{\partial z}\left(a_{11} \frac{\partial}{\partial z} \frac{\mathbf{v} \cdot \mathbf{v}}{2}\right) & -a_{11} \frac{\partial \mathbf{v}}{\partial z} \cdot \frac{\partial \mathbf{v}}{\partial z} \\
& -\left[\frac{\partial}{\partial z}\left(a_{12} \frac{\partial \mathbf{v}}{\partial z}\right) \times \mathbf{v}\right] \cdot \mathbf{k}
\end{aligned}
$$

Thus the tensor element $a_{11}$ is responsible for the diffusion of kinetic energy and the dissipation (first and second terms of the right-hand side), whereas the frictional force $(\partial / \partial z) a_{12}(\partial v / \partial z)$ does (positive or negative) work, if this frictional force is not parallel to the velocity vector.

3. In the context of Reynolds stresses, (19) means that within the Ekman layer the divergence of the Reynolds stress tensor $\left(\partial / \partial x_{i}\right)\left(\left\langle v_{i}^{\prime} v_{k}^{\prime}\right\rangle\right)$ is simplified such that 

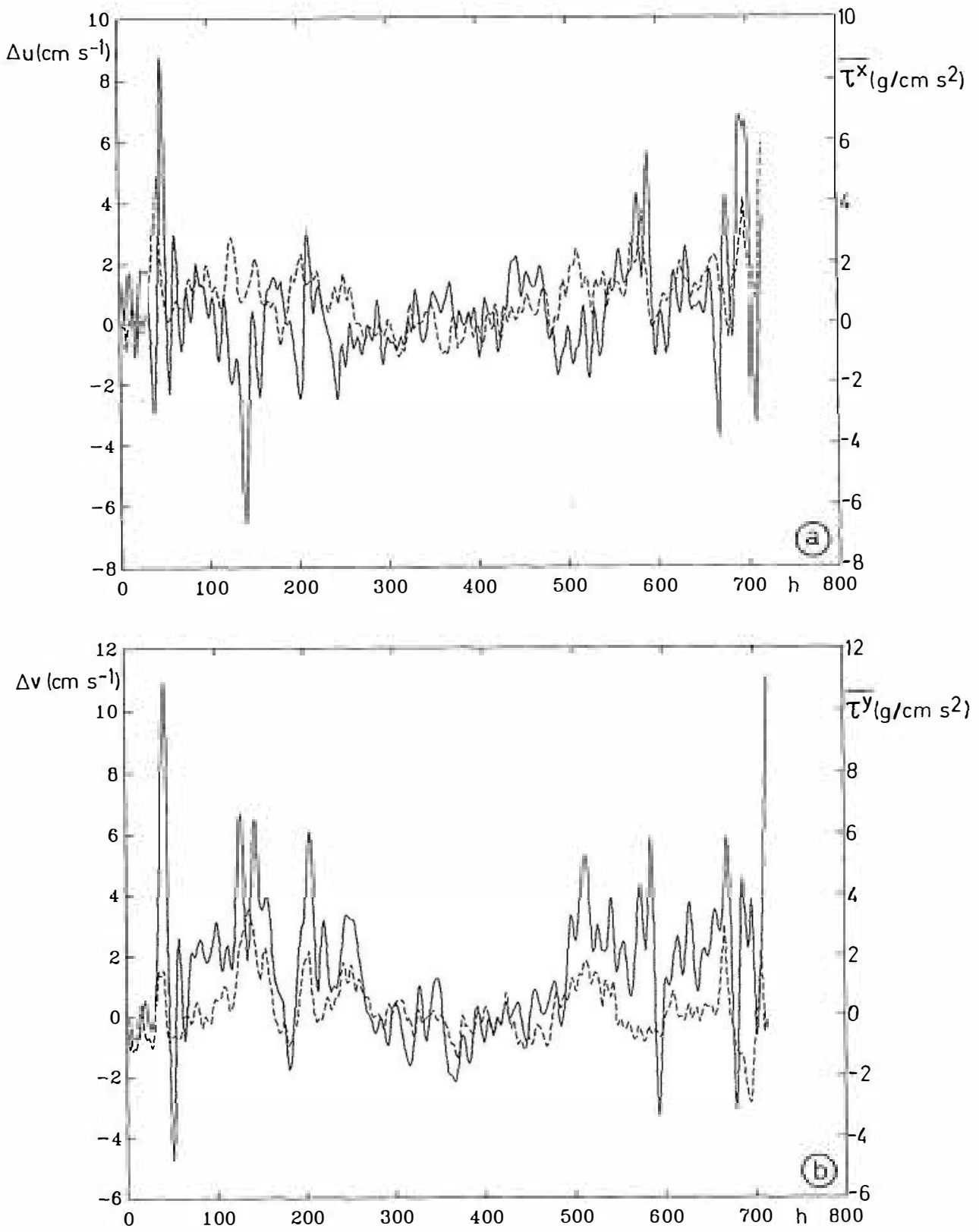

Fig. 18. Difference of Ekman velocities $\Delta u, \Delta v$ (solid line) between $10 \mathrm{~m}$ and $30 \mathrm{~m}$ depth and stresses $\left(\left\langle\tau^{\cdot x}\right\rangle,\left\langle\tau^{y}\right\rangle\right)$ averaged over 15,20 and $25 \mathrm{~m}$ (dashed line), for $(a) x$ component and $(b) y$ component.

$\frac{\partial}{\partial x_{i}}\left\langle v_{i}^{\prime} v_{k}^{\prime}\right\rangle=\left(\begin{array}{c}\frac{\partial\left\langle v_{1}^{\prime} v_{3}^{\prime}\right\rangle}{\partial x_{3}} \\ \frac{\partial\left\langle v_{2}^{\prime} v_{3}^{\prime}\right\rangle}{\partial x_{3}} \\ 0\end{array}\right)=\frac{\partial}{\partial x_{3}} \tau_{E}=\frac{\partial}{\partial x_{3}}\left(A \cdot \frac{\partial \mathbf{v}_{E}}{\partial x_{3}}\right)$

where $x_{3}=z$.

\section{Stresses Not Related to the Wind}

In section 5 we separated the component $\tau_{W}$ from the appare nt stress $\tau$, which is directly related to the wind stress by means of $D_{w}$, and decomposed it into the Ekman stress $\tau_{E}$ and residual $\tau_{R}$, which was interpreted as correction to $\nabla \zeta$. We now consider the remaining part of the stress, which is not related to the local wind stress, $\tau-\tau_{W}$, i.e., the difference between the curves in Figures $13 a$ and $13 b$. This difference is zero at $10-\mathrm{m}$ depth for both $\tau^{x}$ and $\tau^{y}$ as outlined there and increases with increasing depth. As this part of the stress field is not related to the wind, we first check whether or not it varies linearly with depth and thus

TABLE 3. Elements $a_{i k}$ of the Eddy Viscosity Tensor $A$

\begin{tabular}{crrrr}
\hline Depth, m & $a_{11}$ & $a_{12}$ & $a_{21}$ & $a_{22}$ \\
\hline 10 & 71 & 309 & -280 & 124 \\
15 & 340 & 865 & -724 & 439 \\
20 & 1460 & 666 & -396 & 1363 \\
25 & 852 & -440 & 440 & 679 \\
\hline
\end{tabular}




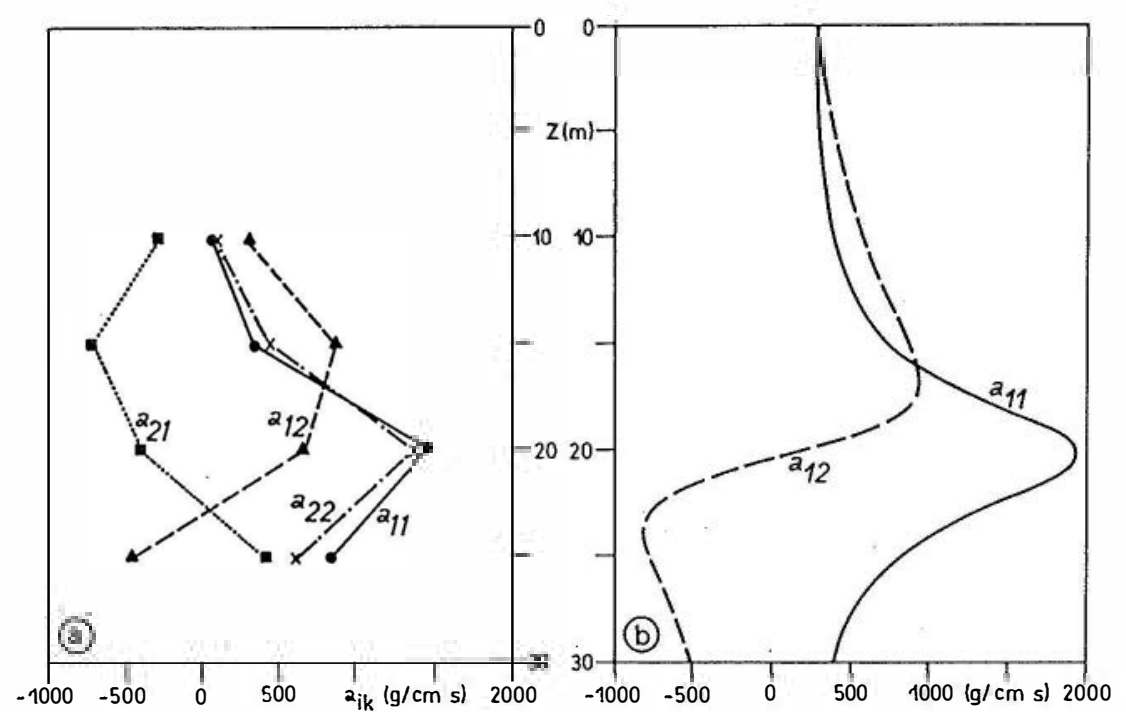

Fig. 19. Elements of the eddy viscosity tensor $A$ as derived from (19), (a) by the method of least squares and $(b)$ according to (21).

can be interpreted as barotropic current. To find an internal relation between these stresses, we relate all stresses arbitrarily to those at $15-\mathrm{m}$ depth. Figure 21 displays these stresses $\tau_{E x}=\tau-\tau_{W}$ and their approximation

$$
\tau_{E x}(z)=D_{E x} \cdot \tau_{E x}(15)
$$

i.e., the dependency of $\tau_{E x}$ from that at $15-\mathrm{m}$ depth. The depth of $15 \mathrm{~m}$ has been chosen because $\tau_{E x}=0$ at $10 \mathrm{~m}$. It turns out that the tensor $D_{E x}$, which describes this relation (Figure 22), can be described to a very high degree of accuracy by the simple linear relations

$$
\begin{array}{ll}
d_{E x 11}=-1.12+0.64 n & d_{E x 12}=-0.28+0.11 n \\
d_{E x 21}=-0.02+0.01 n & d_{E x 22}=-1.45+0.78 n
\end{array}
$$

with $n=0, \cdots, 6(z=n d z, d z=5 \mathrm{~m}, z$ positive downward), which means that the associated currents are barotropic.

In section 5 we interpreted the wind-dependent part of the stress, $\tau_{W}$, which also varied linearly with depth, as a correction to the sea level gradient. The barotropic currents due to $d \tau_{E x} / d z$ are not related to the local wind. However, it is well known that sea level variations in the North Sea are only partially produced by the wind field over the North Sea. The open boundary to the Atlantic Ocean in the north allows external surges to travel into the North Sea, yielding sea level variations along the coast of up to more than a meter [Dietrich et al., 1980]. These variations are not described by the numerical model for the area shown in Figure 1. We therefore interpret the apparent forces $d \tau_{E x} / d z$ as sea level variation due to external sources.

Thus the total barotropic current is produced by $-\boldsymbol{g} \nabla \zeta+$ $d \tau_{R} / d z+d \tau_{E x} / d z$, whereas the Ekman current results from $d \tau_{E} / d z$. In order to compare the barotropic currents with the Ekman currents, Figure 23 depicts the Ekman currents in $10-\mathrm{m}$ and $30-\mathrm{m}$ depth and the total barotropic current, as resulting from the numerical integration of (1) and (2) with the forces mentioned above. Both the Ekman currents and the total barotropic currents are of comparable magnitude, which is the main reason that Ekman currents are difficult to recognize from direct current measurements without a detailed analysis. Also note that very often Ekman currents and barotropic currents nearly cancel each other, which is typical for the central North Sea. This is the reason the drift buoys remain near the position of deployment even during storm periods (Figure 5).

The fact that the barotropic currents and the Ekman currents often appear to be out of face by $180^{\circ}$ is typical for that part of the North Sea. Consider, for example, a wind from north. This piles up water in the southern part of the North Sea and produces a barotropic geostrophic current (under stationary conditions) toward east. The Ekman currents, however, are toward the right of the wind, i.e., toward west. Thus the local barotropic currents and the Ekman currents have a component out of phase by $180^{\circ}$ and are highly correlated. Similar conditions hold for other wind directions.

\section{Modeling the Ekman Layer}

In the preceding sections we were able to separate the Ekman stress $\tau_{E}$ from the barotropic forces. Using these stresses and the wind stress, we can compute the Ekman currents directly from the force field.

The concept of a scalar eddy viscosity is not suitable for modeling the observed currents. However, using the concept of an eddy viscosity tensor, the Ekman stresses can be adequately described by a relation $\tau_{E}=A \cdot \partial \mathbf{v}_{E} / d z$, as shown in Figure 20. Using (21), both descriptions give identical results.

We can conclude this analysis by comparing the observed currents with the numerically computed currents, using $-g \nabla \zeta$ from the numerical model, the barotropic forces $d \tau_{R} / d z$ and $d \tau_{E x} / d z$, which are understood as corrections to the barotropic model, and the Ekman stresses $\tau_{E}$. The results are shown in Figure 24. We are able to reproduce the 

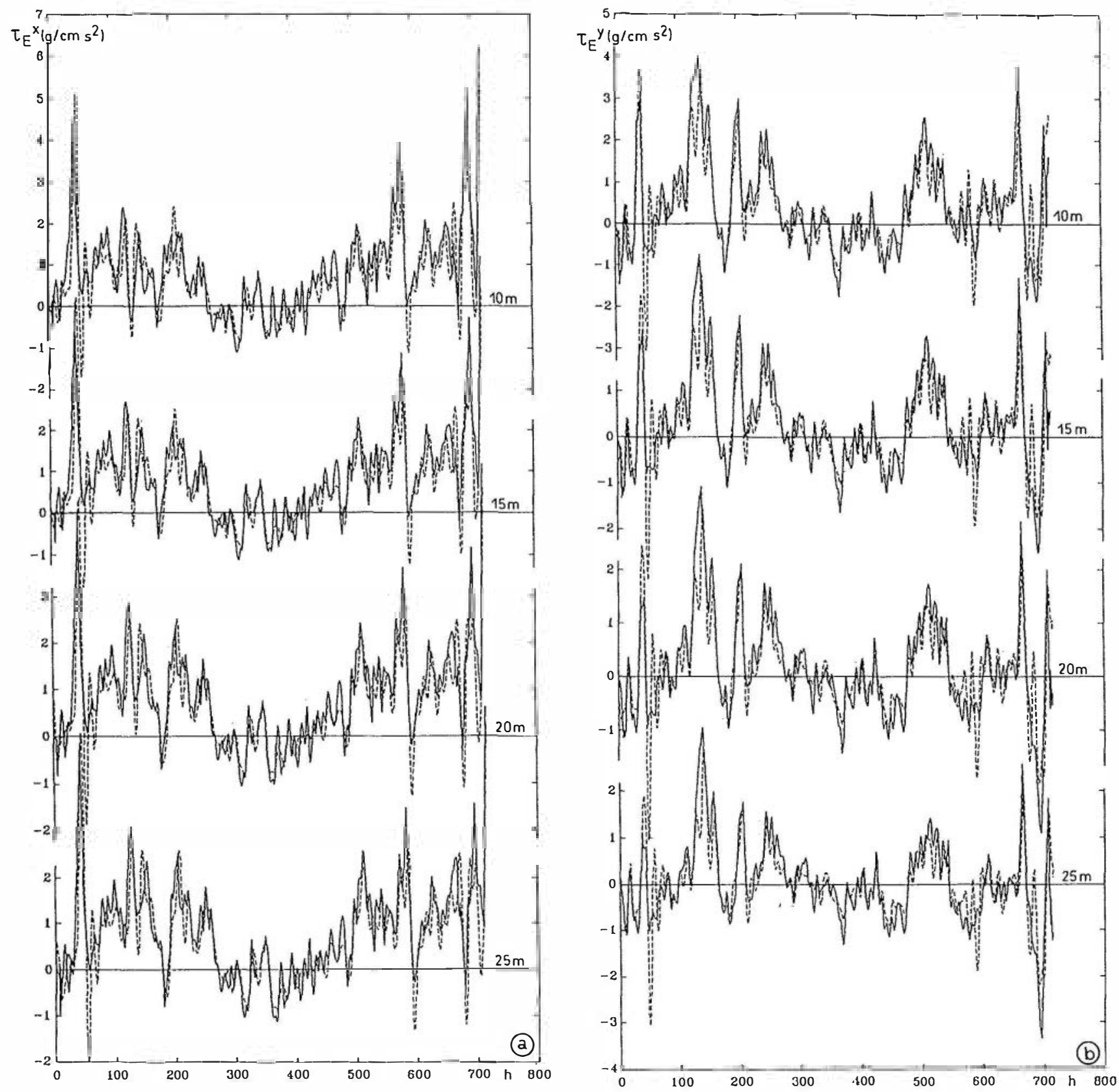

Fig. 20. Comparison between the Ekman stress $\tau_{E}$ (solid line) according to (14) and its approximation (dashed line) with the eddy viscosity tensor (19), for $(a) x$ component and $(b) y$ component.

major fluctuations of the current field within the upper $30 \mathrm{~m}$ of the water column as well as many details of the variations. There is no obvious difference in the quality of description during periods of strong and weak wind velocities. We therefore believe that the Ekman stresses described in section 5 are valid within a large range of wind conditions in homogeneous water.

The description of the Ekman layer by the Ekman stresses seems to be preferable at present compared to a description by the viscosity tensor. The Ekman stresses are related to the wind stress by a simple relationship (equation (14)), where we used the fact that the stress at the surface must equal the wind stress. The depth dependency of the viscosity tensor appears more complicated, and at present it can be derived only from the Ekman tensor. Thus it appears as a secondary quantity, used by tradition. More research is needed on that subject before the complicated vertical structure of this tensor (Figure 19) can be interpreted physically.

\section{CONCLuSIONS}

Satellite-tracked buoys drogued at five different levels within the Ekman layer have been used to test the Ekman theory in the central North Sea. The site has been chosen because (1) it is known to be homogeneous during wintertime, allowing testing of the classical Ekman spiral, (2) relatively good wind data are available for the area of the 

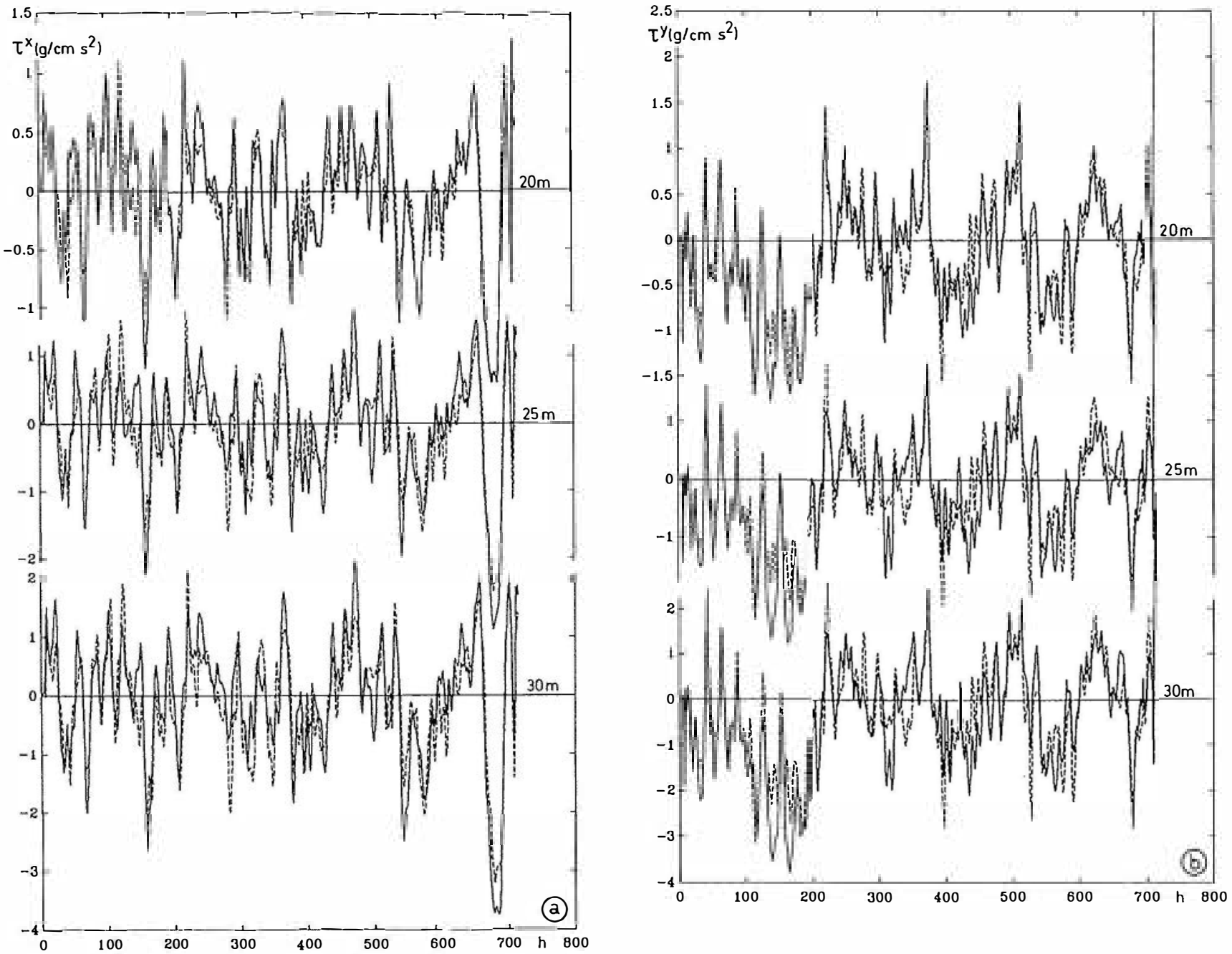

Fig. 21. Remaining stress field $\tau_{E X}=\tau-\tau_{W}$ (solid line) that is not related to the wind stress and its approximation by (30), for $(a) x$ component and $(b) y$ component.

entire North Sea, and (3) model experiments show that the barotropic currents and the Ekman currents partially balance each other, and thus the drifters stay close together over a long time period.

Tides and inertial current have been removed to some extent, and a 4-week-period, during which the buoys stayed within $40 \mathrm{~km}$ has been used to compute the vertical distribution of the apparent stresses from the equations of motion. In this area with homogeneous density, only barotropic and Ekman currents are possible. The barotropic component has been determined with a vertically integrated model of the area and corrected by a residual stress term, which increased linearly with depth. Its vertical derivative can thus be incorporated into the sea level gradients. Another part of the stress that also increased linearly with depth but without being related to the wind field was interpreted as external surges. This interpretation does not exclude the possibility of an increasing stress with depth; we consider our interpretation only as the most likely. The remaining Ekman stress is the dominant part of the total stress and can be directly derived from the wind stress, if this is properly known. One of the outstanding results is that the Ekman stress tensor, which relates the Ekman stress to the wind stress, describes the Ekman currents under all wind conditions that occurred

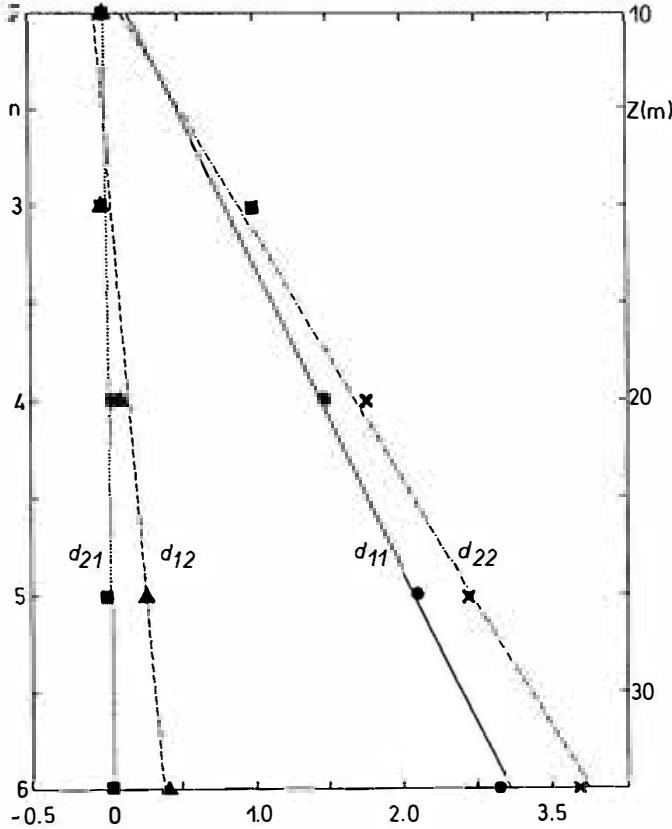

Fig. 22. Depth dependency of $D_{E X}$ : observed values (symbols) and linear relationship (lines). 

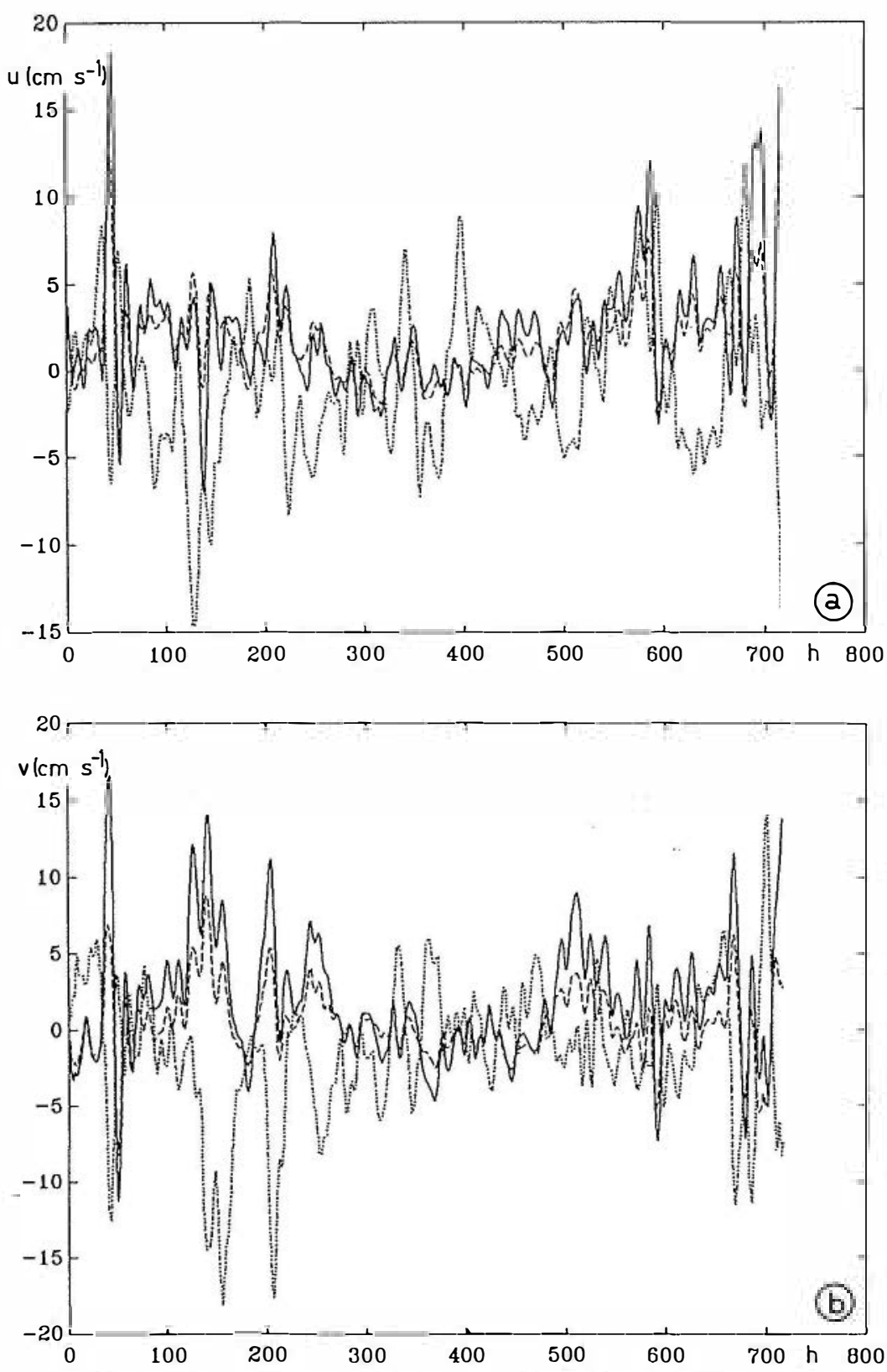

Fig. 23. Ekman currents in $10-\mathrm{m}$ depth (solid line) and 30-m depth (dashed line) and total barotropic current (dotted line), for $(a) u$ component and $(b) v$ component.

during the 4-week period from November 20 to December 19, 1991. The mean observed velocities within the upper 30 $\mathrm{m}$ were $\langle u\rangle=1.4 \mathrm{~cm} \mathrm{~s}^{-1}$ and $\langle v\rangle=-0.7 \mathrm{~cm} \mathrm{~s}^{-1}$. The modeled mean velocities are $\langle u\rangle=1.3 \mathrm{~cm} \mathrm{~s}^{-1}$ and $\langle v\rangle=$ $-0.9 \mathrm{~cm} \mathrm{~s}^{-1}$. The corresponding rms velocities are $u^{\prime}=4.5$ $\mathrm{cm} \mathrm{s}^{-1}, v^{\prime}=5.4 \mathrm{~cm} \mathrm{~s}^{-1}$ (observed) and $u^{\prime}=4.4 \mathrm{~cm} \mathrm{~s}^{-1}$, $v^{\prime}=3.8 \mathrm{~cm} \mathrm{~s}^{-1}$ (modeled).

It turns out that the original assumption of Ekman to describe friction by a constant eddy viscosity is not appropriate. However, an eddy viscosity tensor is able to simulate the observed stresses remarkably well but it is simply a secondary quantity derived from the Ekman stress tensor and the Ekman dynamic. We therefore prefer the concept of stresses at present. The Ekman layer is then described under linear conditions for a homogeneous fluid by a body force according to

$$
\frac{\partial \mathbf{v}}{\partial t}+\mathbf{f} \times \mathbf{v}=-\boldsymbol{g} \nabla \zeta+\frac{1}{\rho_{0}} \frac{d D_{E}}{d z} \cdot \tau_{0}
$$

where $D_{E}$ is the Ekman tensor and $\tau_{0}$ is the wind stress. From our observations, $D_{E}$ is known in the upper $30 \mathrm{~m}$ only. However, inspection of Figure 14 suggests that both $d_{E 11}$ and $d_{E 12}$ approach a constant value at some depth, which means that the body force in (31) becomes zero. Thus the forcing is limited to the upper layers.

As was mentioned in section 2, only 4 weeks of a 4-month experiment were used for this analysis. The remaining 3 months of observations will be used to test whether the results obtained are also able to describe the drift of the buoys after they are scattered over a larger area. 

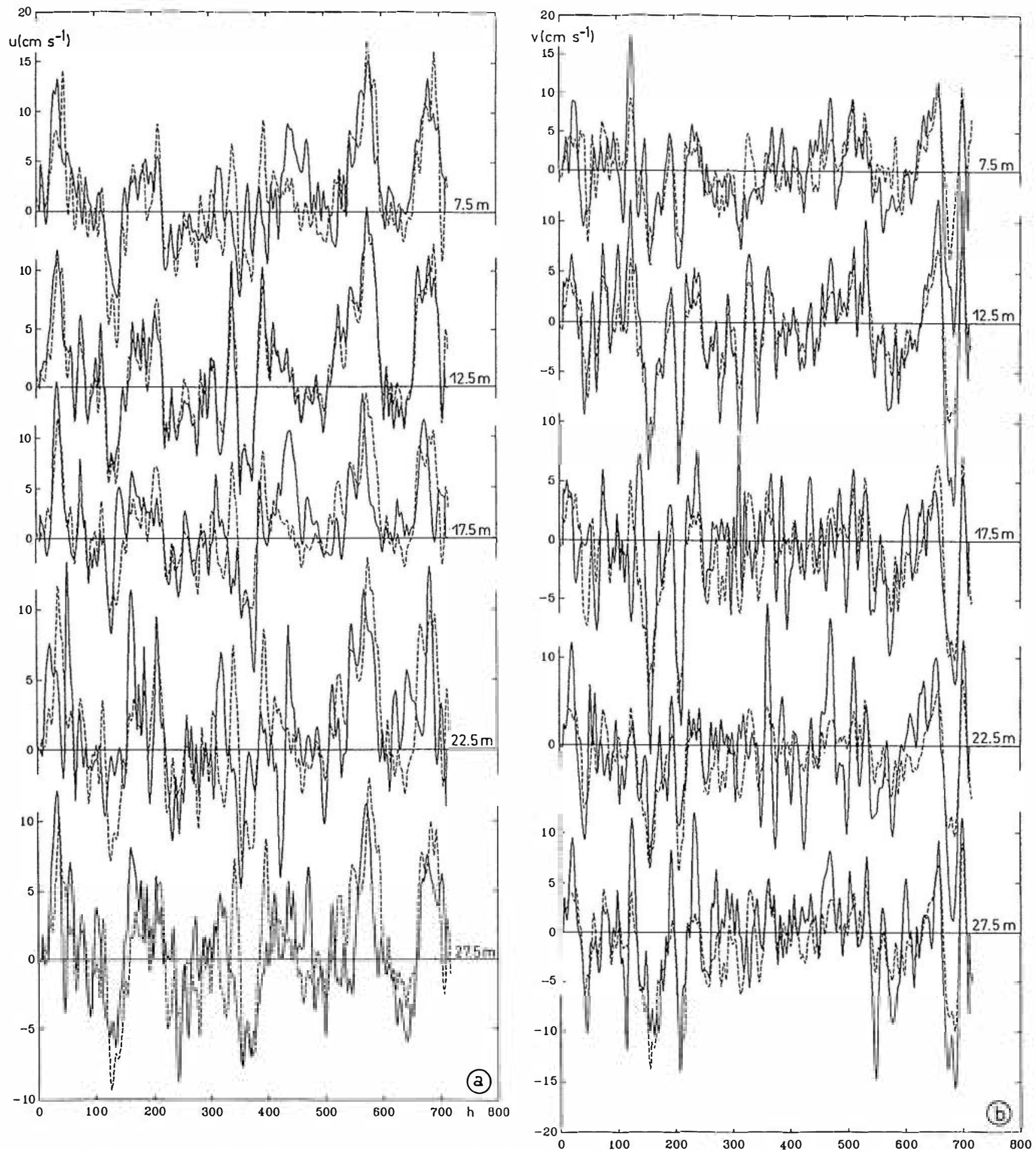

Fig. 24. Observed currents (solid line) and numerically computed currents (dashed line) in 7.5-m, 12.5-m, 17.5-m, 22.5-m, and 27.5 - $\mathrm{m} \mathrm{depth}$, for $(a) u$ component and $(b) v$ component.

The results of this winter experiment are supposed to be typical for mid and high latitudes during the winter season. The water depth of $80 \mathrm{~m}$ in the area should have no major influence on the results, as Figure 19 suggests that the viscosity tensor decreases rapidly between 20 -m depth and the bottom. We therefore assume that the results are not influenced by the seafloor. However, it will be hard to prove that they hold generally in the open ocean within a deep mixed layer. The main difficulty in analyzing the Ekman stresses results from the need to separate the Ekman currents from the remaining ones. In the open ocean the instantaneous current field is dominated by the eddies; their exact location, growth, and decay cannot be modeled realistically at present. Thus a similar approach to determine the Ekman stresses in the open ocean is unlikely to be successful. Drift buoys drogued at different depths, will not stay together in the open ocean owing to dispersion by the eddy field. Measurements by moored 
instruments are extremely difficult to perform in the harsh environment of the upper layers. Using the difference between shipboard acoustic Doppler current profiler measurements and geostrophic velocities from conductivitytemperature-depth data [Chereskin and Roemmich, 1991], however, may give more information at least under relatively calm wind conditions.

The consequences of the present results for numerical models should be examined. Most models of the windgenerated ocean circulation accept the Ekman concept of a scalar eddy viscosity. Moreover, the values used in these models are sometimes orders of magnitude smaller than those found in the present study. The poor performance of models at high latitude may be due to a wrong Ekman dynamic. Furthermore, if the Ekman currents penetrate below the base of the mixed layer, as is indicated by Chereskin and Roemmich [1991], the Ekman heat transport may be modified considerably.

Ekman pumping is the principal mechanism driving the large-scale wind-induced circulation in the ocean. Contrary to the classical description of the Ekman layer, the vorticity associated with (31) is given by

$$
\begin{aligned}
\frac{\partial \zeta_{\mathrm{rel}}}{\partial t}+f\left(\frac{\partial u}{\partial x}+\frac{\partial v}{\partial y}\right)+\beta v & =\frac{1}{\rho_{0}} \frac{d\left(d_{E 11}\right)}{d z}\left(\frac{\partial \tau_{0}^{y}}{\partial x}-\frac{\partial \tau_{0}^{x}}{\partial y}\right) \\
& -\frac{1}{\rho_{0}} \frac{d\left(d_{E 12}\right)}{d z}\left(\frac{\partial \tau_{0}^{x}}{\partial x}+\frac{\partial \cdot \tau_{0}^{y^{\prime}}}{\partial y}\right)
\end{aligned}
$$

and the Ekman pumping at the bottom of the mixed layer (or wherever the Ekman tensor becomes constant) is given by

$$
\begin{gathered}
W(-h)=\frac{1}{\rho_{0} f}\left\{\left[1-d_{E 11}(-h)\right]\left(\frac{\partial \tau_{0}^{y}}{\partial x}-\frac{\partial \tau_{0}^{x}}{\partial y}\right)+d_{E 12}(-h)\right. \\
\left.\cdot\left(\frac{d \tau_{0}^{x}}{d x}+\frac{d \tau_{0}^{y}}{\partial y}\right)\right\}
\end{gathered}
$$

under stationary conditions on the $f$ plane.

Thus the large-scale circulation is not only driven by the curl of the wind stress but also by its divergence. Inspection of the stress field over the North Atlantic [Isemer and Hasse, 1987] shows that in some areas in mid and high latitudes the wind stress divergence and the curl are of equal amplitude.

We finally suggest reexamining the usefulness of slab models. A crude inspection of the velocity records (Figure 6) supports such an approach because the observed currents within the upper $30 \mathrm{~m}$ are fairly uniform. However, after the barotropic currents are eliminated, a clear Ekmantype spiral emerges. In the deep ocean the baroclinic currents mask the Ekman currents. The baroclinic currents also are nearly uniform above the main thermocline and may be a major contributor to the total current within the mixed layer. Generally speaking, we may have been misled by numerical models, which use low values of eddy viscosity and therefore produce large Ekman currents. These observations show that the eddy viscosity tensor is of the order of 1000 and, consequently, Ekman currents are small in homogeneous water. They may not be, in general, the dominant currents in the mixed layer. Part of the uniform barotropic or nearly uniform baroclinic currents in the mixed layer may well be misinterpreted as Ekman currents. Slab models are based on a linear decreasing stress with depth, making $d \tau / d z$ constant and thus making the current uniform. Such a stress distribution is not supported by our analysis.

\section{REFERENCES}

Bitterman, D. S., and D. V. Hansen, The design of a low cost tropical drifter buoy, paper presented at Marine Data Systems International Symposium, Mar. Technol. Soc., New Orleans, La., April 30 to May 2, 1986.

Blake, R. A., The dependence of wind stress on wave height and wind speed. J. Geophys. Res., 96, 20,531-20,545, 1991.

Böning, C. W., and P. Herrmann, On the annual cycle of poleward heat transport in the ocean: Results from high resolution modeling of the North and equatorial Atlantic, J. Phys. Oceanogr., in press, 1993.

Chereskin, T. K. and D. Roemmich, A comparison of measured and wind-derived Ekman transport at $11^{\circ} \mathrm{N}$ in the Atlantic Ocean, $J$. Phys. Oceanogr., 21, 869-878, 1991.

Defant, A., Physical Oceanography, vol. 1, pp. 415--423, Pergamon, New York, 1960.

Dietrich, G., K. Kalle, W. Krauss, and G. Siedler, General Oceanography, vol. 1, 626 pp., John Wiley, New York, 1980.

Ekman, V. W., Om jordrotationens inverkam pa windströmmar i hafvet, Nytt Mag. Naturvidenskab., 2, 40(1), 1-52, 1902.

Ekman, V. W., On the influence of the Earth's rotation on oceancurrents, Ark. Mat. Astron. Fys., 2(11), 1-36, 1905.

Ertel, H., Tensorielle Theorie der Turbulenz, Ann. Hydrogr. Mar. Meteorol., 65, 193-205, 1937.

Fischer, G., Ein numerisches Verfahren zur Errechnung von Windstau und Gezeiten in Randmeeren, Tellus, 11, 60--76, 1959.

Flament, P., L. Armi, and L. Washburn, The evolution of an upwelling filament, J. Geophys. Res., 90, 11,765-11,778, 1985.

Gonella, J., Observation de la spirale d'Ekman en Méditerranée occidentale, C. R. Acad. Sci. Ser. B, 266, 205-208, 1968.

Huang, N. E., On surface drift currents in the ocean, J. Fluid. Mech., 91, 191-208, 1979.

Ichiye, T., Upper ocean boundary-layer flow determined by dye diffusion, Phys. Fluids, 10, suppl., 270-277, 1967.

Isemer, H.-J., and L. Hasse, The Bunker Climate Atlas of the North Atlantic Ocean, vol. 2, Air-Sea Interactions, 252 pp., SpringerVerlag, New York, 1987.

Katz, B., R. Gerard, and M. Costin, Response of dye tracers to sea surface conditions, J. Geophys. Res., 70, 5505-5513, 1965.

Kondo, J., Y. Sasano, and T. Ishii, On wind-driven current and temperature profiles with diurnal period in the oceanic planetary boundary layer, J. Phys. Oceanogr., 9, 360--372, 1979.

Large, W. G., and S. Pond, Open ocean momentum flux measurements in moderate to strong winds, J. Phys. Oceanogr., II, 324--336, 1981.

Leibovich, S., and K. Radhakrishnan, On the evolution of the system of wind drift currents and Langmuir circulations in the ocean, 1, Theory and averaged current, J. Fluid Mech., 79, 715-743, 1977.

Luthardt, H., and L. Hasse, The relationship between pressure field and surface wind in the German Bight area at high wind speeds, in North Sea Dynamics, edited by J. Sündermann and W. Lenz, pp. 340--348, Springer-Verlag, New York, 1983.

Madsen, O. S., A realistic model of the wind-induced Ekman boundary layer, J. Phys. Oceanogr., 7, 248-255, 1977.

McNally, G. J., and W. B. White, Wind-driven flow in the mixedlayer observed by drifting buoys during autumn-winter in the mid latitude North Pacific, J. Phys. Oceanogr., 15, 684--694, 1985.

Nansen, F., 1902: Oceanography of the North Polar Basin, The Norwegian North Polar Expedition 1893-1896. Scientific Results, Vol. III, No. 9, Kristiania.

Neumann, G., and W. J. Pierson, Principles of Physical Oceanography, Prentice-Hall, Englewood Cliffs, N. J., 1964.

Price, J. F., R. A. Weller, and R. Pinkel, Diurnal cycling: Observa- 
tions and models of the upper ocean response to diurnal heating, cooling, and wind mixing, J. Geophys. Res., 91, 8411--8427, 1986. Price, J. F., R. A. Weller, and R. R. Schudlich, Wind-driven ocean currents and Ekman transport, Science, 238, 1534-1538, 1987.

Smith, J., R. Pinkel, and R. A. Weller, Velocity structure in the mixed layer during MILDEX, J. Phys. Oceanogr., 17, 425-439, 1987.

Stacey, M. W., S. Pond, and P. H. LeBlond, A wind-forced Ekman spiral as a good statistical fit to low-frequency currents in a coastal strait, Science, 233, 470-472, 1986.

Thorade, H., Die Geschwindigkeit von Triftströmungen und die Ekmansche Theorie, Ann. Hydrogr. Mar. Meteorol., 42, 379-391, 1914.
Weller, R. A., Observations of the velocity response to wind forcing in the upper ocean, J. Geophys. Res., 86, 1969-1977, 1981.

Weller, R. A., J. Marra, J. Price, E. A. Francis, and D. C. Boardman, Three-dimensional flow in the upper ocean, Science, 227, 1552-1556, 1985.

W. Krauss, Institut für Meereskunde an der Universität Kiel, Düsternbrooker Weg 20, D-24105 Kiel 1, Germany.

\section{(Received October 16, 1992;} revised June 22, 1993; accepted June 22, 1993.) 